\title{
Interpolation and Scattered Data Fitting on Manifolds using Projected Powell-Sabin Splines
}

\author{
Oleg Davydov* and Larry L. Schumaker ${ }^{\dagger}$
}

August 31, 2007

\author{
Dedicated to Professor M.J.D. Powell \\ on the occasion of his 70th birthday
}

\begin{abstract}
We present methods for either interpolating data or for fitting scattered data on a two-dimensional smooth manifold $\Omega$. The methods are based on a local bivariate Powell-Sabin interpolation scheme, and make use of a family of charts $\left\{\left(U_{\xi}, \phi_{\xi}\right)\right\}_{\xi \in \Omega}$ satisfying certain conditions of smooth dependence on $\xi$. If $\Omega$ is a $C^{2}$-manifold embedded into $\mathbb{R}^{3}$, then projections into tangent planes can be employed. The data fitting method is a two-stage method. We prove that the resulting function on the manifold is continuously differentiable, and establish error bounds for both methods for the case when the data are generated by a smooth function.
\end{abstract}

\section{Introduction}

Let $\Omega$ be a 2 -dimensional smooth manifold. For simplicity we assume that $\Omega$ is compact and has no boundary. Suppose we are given the values of a

*Department of Mathematics, University of Strathclyde, 26 Richmond Street, Glasgow G1 1XH, Scotland. Partially supported by the Edinburgh Mathematical Society Research Support Fund.

${ }^{\dagger}$ Department of Mathematics, Vanderbilt University, Nashville, TN 37240. 
(possibly unknown) smooth function $f$ defined on $\Omega$ at a set of points $X$ on $\Omega$. Our aim is to construct a function $s$ defined on $\Omega$ that approximates $f$. This problem arises frequently in practice, see Remark 8.1, but there do not seem to be many methods available for general manifolds. Several methods have been developed for the case when $\Omega$ is the sphere, see Remark 8.2.

Our approach to solving this problem is as follows. Suppose we have an atlas $\Phi=\left\{\left(U_{\xi}, \phi_{\xi}\right)\right\}_{\xi \in \Omega}$ for $\Omega$, where for each $\xi \in \Omega, U_{\xi}$ are open sets on $\Omega$ containing $\xi$, and $\phi_{\xi}$ are mappings of $U_{\xi}$ into $\mathbb{R}^{2}$. We assume that the $\phi_{\xi}$ depend smoothly on $\xi$ in a way to be described in Definition 3.1. Then for each $\xi \in \Omega$, we map the data locations into $\phi_{\xi}\left(U_{\xi}\right) \subset \mathbb{R}^{2}$, and use a local bivariate Powell-Sabin spline to compute the value $s(\xi)$ of the approximating function $s$. This approach is related to methods introduced by Demjanovich $[11,12]$ and Pottmann [29], see Remarks 8.3 and 8.4.

The paper is organized as follows. In Section 2 we describe the classical bivariate piecewise quadratic spline interpolant by Powell and Sabin [30], and prove that it depends smoothly on the vertex locations and the data. In Section 3 we introduce some basic concepts and notation, including atlases, gradients, Sobolev spaces, and triangulations on manifolds. In view of the Poincaré-Hopf index theorem, local parametrizations defined by the charts $\left(U_{\xi}, \phi_{\xi}\right)$ in general cannot be smooth functions of $\xi$, see Remark 8.7. Therefore, we introduce a weaker concept of smoothness whereby $\phi_{\zeta}$ for $\zeta$ close to $\xi$ may be adjusted by local rotations or rotoinversions (see Definition 3.1). In Section 4 we present a method for constructing an interpolant to data on an arbitrary smooth 2-dimensional manifold $\Omega$, assuming we are also given values for the gradients at each of the data locations in $X$. We show that the method produces a $C^{1}$ function on $\Omega$, and give an error bound for how well it approximates smooth functions. In Section 5 we describe a two-stage scattered data fitting method which is more appropriate than interpolation for large data sets, and for highly irregularly distributed or noisy data. This method does not require the knowledge of the gradients. In the next section we give an error bound for this method. In Section 7 we specialize to the case where the manifold is embedded in $\mathbb{R}^{3}$. In particular, we show how to explicitly construct an atlas with the required properties using local projections into tangent planes and discuss certain simplifications in the algorithms. More details on our method used with this specific atlas can be found in our paper [9], which also gives numerical examples for both the sphere and for certain ring-type manifolds. We conclude the paper with remarks and references. 

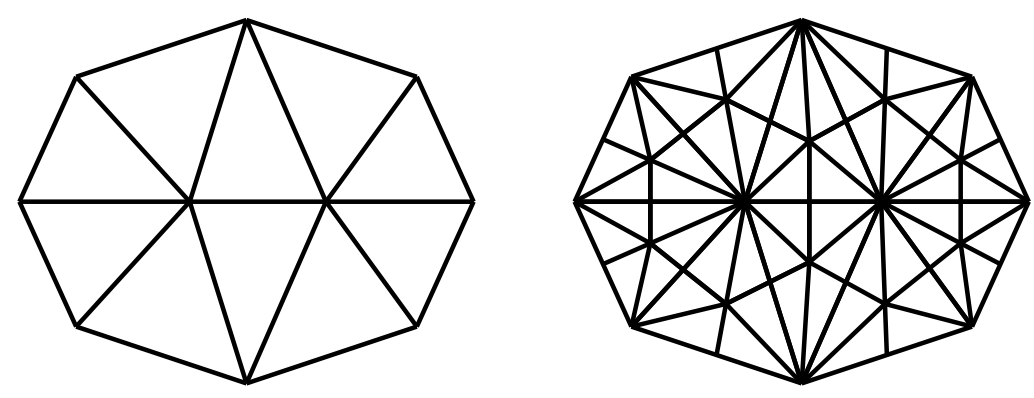

Figure 1: A triangulation $\triangle$ and its Powell-Sabin refinement $\triangle_{P S}$.

\section{The Powell-Sabin Spline Interpolant}

We begin by recalling some facts about certain $C^{1}$ quadratic spline spaces introduced in [30]. Let $\triangle$ be a regular triangulation of a polygonal domain $G$ in $\mathbb{R}^{2}$, and let $\left\{v_{i}\right\}_{i=1}^{n}$ be its set of vertices, where $v_{i}:=\left(x_{i}, y_{i}\right)$. We write $\triangle_{P S}$ for the well-known Powell-Sabin refinement of $\triangle$, where the split point $v_{T}$ in each triangle $T$ is taken to be its incenter see Definition 4.18 of [21]. Thus, $\triangle_{P S}$ is obtained from $\triangle$ by connecting the incenter of each triangle $T$ to the incenters of all triangles in $\triangle$ that share an edge with $T$, and to the vertices of $T$. If $T$ has any edges on the boundary of $G$, then we also connect $v_{T}$ with the midpoints of such edges. Figure 1 shows a triangulation $\triangle$ and its associated Powell-Sabin refinement, see also Figure 3. It is well known that for any real numbers $\left\{z_{i}, z_{i}^{x}, z_{i}^{y}\right\}_{i=1}^{n}$, there exists a unique spline $s$ in the space $\mathcal{S}_{2}^{1}\left(\triangle_{P S}\right)$ of all $C^{1}$ piecewise quadratic functions with respect to $\triangle_{P S}$ such that

$$
s\left(v_{i}\right)=z_{i}, D_{x} s\left(v_{i}\right)=z_{i}^{x}, D_{y} s\left(v_{i}\right)=z_{i}^{y}, \quad i=1, \ldots, n,
$$

where $D_{x}, D_{y}$ stand for the $x$ - and $y$-derivatives, respectively.

It is also known that the value $s(u)$ at a point $u \in G$ depends locally on the data $z=\left(z_{1}, z_{1}^{x}, z_{1}^{y}, \ldots, z_{n}, z_{n}^{x}, z_{n}^{y}\right)$. In particular, if $u$ lies in the triangle $T \in \triangle$, then $s(u)$ is uniquely determined by the data at the three vertices of $T$.

Clearly, the value $s(u)$ is also a function of the locations of the vertices in the set $\left\{v_{i}\right\}_{i=1}^{n}$. So to be precise we should write $s(V, z, u)$ to show the dependence on $V:=\left(v_{1}, \ldots, v_{n}\right), z$ and $u$. Suppose $\triangle^{\varepsilon}$ is a triangulation obtained from $\triangle$ by perturbing the vertices by a small amount. Clearly, a 
sufficiently small perturbation does not produce any degenerate or overlapping triangles in $\triangle^{\varepsilon}$. We write $V^{\varepsilon}$ for the perturbed vertices, and $\triangle_{P S}^{\varepsilon}$ for the corresponding Powell-Sabin refinement of $\triangle^{\varepsilon}$.

Theorem 2.1. $s(V, z, u)$ is a $C^{1}$ function of the parameters $V, z, u$.

Proof. It is well known that the Powell-Sabin spline is shift-invariant in the sense that

$$
s(V-w, z, u-w)=s(V, z, u), \quad \text { for any } w \in \mathbb{R}^{2},
$$

see [30]. This implies that $s(V, z, u)=s\left(V-u+u_{0}, z, u_{0}\right)$ for any fixed $u_{0} \in \mathbb{R}^{2}$. Moreover, since $\mathcal{S}_{2}^{1}\left(\triangle_{P S}\right)$ is a linear space, $s(V, z, u)$ is a linear function of $z$, that is

$$
s(V, z, u)=\sum_{i=1}^{n} z_{i} s\left(V, \epsilon_{i}, u\right)+z_{i}^{x} s\left(V, \epsilon_{i}^{x}, u\right)+z_{i}^{y} s\left(V, \epsilon_{i}^{y}, u\right),
$$

where $\epsilon_{i}, \epsilon_{i}^{x}, \epsilon_{i}^{y} \in \mathbb{R}^{3 n}$ are the corresponding unit vectors. Hence, the theorem will follow if we hold $u$ and $z$ fixed and show that $s(V, z, u)$ is continuously differentiable as a function of $V$.

We consider three cases depending on whether $u$ lies in the interior of a triangle in $\triangle_{P S}$, in the interior of an edge of a triangle, or at a vertex of $\triangle P S$.

Case 1: Suppose $u$ lies in the interior of a triangle $t \in \triangle_{P S}$. Then for any sufficiently small perturbation of the vertices $V, u$ also lies in the interior of the perturbed triangle $t^{\varepsilon}$. Let

$$
q^{\varepsilon}:=\sum_{i+j+k=2} c_{i j k}^{\varepsilon} B_{i j k}^{\varepsilon}
$$

be the quadratic polynomial that coincides with the spline $s^{\varepsilon}:=s\left(V^{\varepsilon}, z, \cdot\right)$ on $t^{\varepsilon}$, where $B_{i j k}^{\varepsilon}$ are the quadratic Bernstein basis polynomials associated with $t^{\varepsilon}$, see [21]. Since $B_{i j k}^{\varepsilon}=\frac{2}{i ! j ! k !} b_{1}^{i} b_{2}^{j} b_{3}^{k}$, where $b_{1}, b_{2}, b_{3}$ are the barycentric coordinates relative to $t^{\varepsilon}$, the values $B_{i j k}^{\varepsilon}(u)$ are analytic functions of the coordinates of the vertices of $t^{\varepsilon}$. Moreover, c.f. the formulae in Theorem 6.11 of [21], the $c_{i j k}^{\varepsilon}$ are also analytic functions of the coordinates of the vertices of $\triangle_{P S}^{\varepsilon}$. Since the incenter of a triangle is an analytic function of its vertices, the vertices of $\triangle_{P S}^{\varepsilon}$ are analytic functions of $V^{\varepsilon}$, and we conclude that $s(V, z, u)$ is an analytic function of $V$ in this case. 
For later use, we observe that for any vertex $w$ of $\triangle_{P S}, s(V, z, w)$ is an analytic function of $V$. Indeed, denote by $w^{\varepsilon}$ the perturbed version of $w$, and choose a triangle $t^{\varepsilon} \in \triangle_{P S}^{\varepsilon}$ attached to $w^{\varepsilon}$. Then $s\left(V^{\varepsilon}, z, w^{\varepsilon}\right)=c_{200}^{\varepsilon}$ if the vertices of $t^{\varepsilon}$ are numbered accordingly. As mentioned above, $c_{200}^{\varepsilon}$ is an analytic function of the vertices.

Case 2: Suppose $u$ lies in the interior of an edge $e$ of $\triangle_{P S}$ shared by two triangles $t_{1}, t_{2} \in \triangle_{P S}$. In this case, a small perturbation $V^{\varepsilon}$ of the vertices $V$ will leave $u$ in the interior of $t_{1}^{\varepsilon} \cup t_{2}^{\varepsilon}$. Let $q_{1}^{\varepsilon}$ be the quadratic polynomial defined on $t_{1}^{\varepsilon}$ as in Case 1. Then for $(x, y) \in t_{1}^{\varepsilon} \cup t_{2}^{\varepsilon}$,

$$
s^{\varepsilon}(x, y)=q_{1}^{\varepsilon}(x, y)+\alpha^{\varepsilon} \psi^{\varepsilon}(x, y),
$$

where

$$
\psi^{\varepsilon}(x, y)= \begin{cases}0, & \text { if }(x, y) \in t_{1}^{\varepsilon}, \\ \ell_{\varepsilon}^{2}(x, y), & \text { if }(x, y) \in t_{2}^{\varepsilon},\end{cases}
$$

and $\ell_{\varepsilon}(x, y)$ is a linear function that vanishes on the line containing $e$, and is positive in the interior of $t_{2}^{\varepsilon}$. Then $\psi^{\varepsilon}(x, y)$ is the composition of $\ell_{\varepsilon}(x, y)$ with the univariate truncated power function

$$
\xi_{+}^{2}= \begin{cases}0, & \text { if } \xi \leq 0 \\ \xi^{2}, & \text { if } \xi>0 .\end{cases}
$$

Suppose $\ell_{\varepsilon}$ is chosen in the form $\ell_{\varepsilon}(x, y)=x \cos b+y \sin b+c$ so that it is positive for $(x, y) \in t_{2}^{\varepsilon}$. Clearly, $b$ and $c$ are analytic functions of the location of the vertices. Since $\xi_{+}^{2}$ is a $C^{1}$ function, it follows that $\psi^{\varepsilon}(x, y)$ is a $C^{1}$ function of $x, y$ and $V^{\varepsilon}$. We claim that $\alpha^{\varepsilon}$ is an analytic function of $V^{\varepsilon}$. To see this, let $w^{\varepsilon}$ be the vertex of $t_{2}^{\varepsilon}$ not on the edge $e$. Then the condition $s\left(V^{\varepsilon}, z, w^{\varepsilon}\right)=q_{1}^{\varepsilon}\left(w^{\varepsilon}\right)+\alpha^{\varepsilon} \ell_{\varepsilon}^{2}\left(w^{\varepsilon}\right)$ implies

$$
\alpha^{\varepsilon}=\frac{s\left(V^{\varepsilon}, z, w^{\varepsilon}\right)-q_{1}^{\varepsilon}\left(w^{\varepsilon}\right)}{\ell_{\varepsilon}^{2}\left(w^{\varepsilon}\right)} .
$$

This expression is an analytic function of the vertices $V^{\varepsilon}$, and we conclude that $s(V, z, u)$ is a $C^{1}$ function of $V$ in this case.

Case 3: Suppose $u$ coincides with a vertex $w$ of $\triangle_{P S}$. In this case if $V^{\varepsilon}$ is a sufficiently small perturbation of $V$, then $u$ will lie in the cell $\mathcal{C}^{\varepsilon}=\cup_{\ell=1}^{m} t_{\ell}^{\varepsilon}$ formed by the set $\left\{t_{\ell}^{\varepsilon}\right\}_{\ell=1}^{m}$ of all triangles in $\triangle_{P S}^{\varepsilon}$ attached to the perturbed vertex $w^{\varepsilon}$, numbered in counterclockwise order. For $\ell=1, \ldots, m$, let

$$
q_{\ell}^{\varepsilon}:=\sum_{i+j+k=2} c_{i j k}^{\varepsilon, \ell} B_{i j k}^{\varepsilon, \ell}
$$


be the quadratic polynomial that coincides with the spline $s\left(V^{\varepsilon}, z, \cdot\right)$ on $t_{\ell}^{\varepsilon}$, where $B_{i j k}^{\varepsilon, \ell}$ are the quadratic Bernstein basis polynomials associated with $t^{\varepsilon, \ell}$. Assume that the vertices of $t^{\varepsilon, \ell}$ are numbered such that $w^{\varepsilon}$ is the first. Let $L\left(V^{\varepsilon}, \cdot\right)$ be the linear Taylor polynomial for $s\left(V^{\varepsilon}, z, \cdot\right)$ at $w^{\varepsilon}$. It is not difficult to see that for any $\ell=1, \ldots, m$,

$$
\begin{aligned}
L\left(V^{\varepsilon}, \cdot\right)= & c_{200}^{\varepsilon, \ell} B_{200}^{\varepsilon, \ell}+c_{110}^{\varepsilon, \ell} B_{110}^{\varepsilon, \ell}+c_{101}^{\varepsilon, \ell} B_{101}^{\varepsilon, \ell}+\left(2 c_{110}^{\varepsilon, \ell}-c_{200}^{\varepsilon, \ell}\right) B_{020}^{\varepsilon, \ell} \\
& +\left(c_{110}^{\varepsilon, \ell}+c_{101}^{\varepsilon, \ell}-c_{200}^{\varepsilon, \ell}\right) B_{011}^{\varepsilon, \ell}+\left(2 c_{101}^{\varepsilon, \ell}-c_{200}^{\varepsilon, \ell}\right) B_{002}^{\varepsilon, \ell},
\end{aligned}
$$

which shows that $L\left(V^{\varepsilon}, u\right)$ is an analytic function of the vertices. Therefore it suffices to show that the function

$$
d\left(V^{\varepsilon}\right):=s\left(V^{\varepsilon}, z, u\right)-L\left(V^{\varepsilon}, u\right)
$$

is continuously differentiable at $V^{\varepsilon}=V$. Clearly,

$$
d\left(V^{\varepsilon}\right)=\tilde{c}_{020}^{\varepsilon, \ell} B_{020}^{\varepsilon, \ell}(u)+\tilde{c}_{011}^{\varepsilon, \ell} B_{011}^{\varepsilon, 1}(u)+\tilde{c}_{002}^{\varepsilon, \ell} B_{002}^{\varepsilon, \ell}(u),
$$

where $\ell$ is such that $u \in t_{\ell}^{\varepsilon}$, and

$$
\begin{aligned}
& \tilde{c}_{020}^{\varepsilon, \ell}:=c_{020}^{\varepsilon, \ell}-2 c_{110}^{\varepsilon, \ell}+c_{200}^{\varepsilon, \ell}, \\
& \tilde{c}_{011}^{\varepsilon, \ell}:=c_{011}^{\varepsilon, \ell}-c_{110}^{\varepsilon, \ell}-c_{101}^{\varepsilon, \ell}+c_{200}^{\varepsilon, \ell}, \\
& \tilde{c}_{002}^{\varepsilon, \ell}:=c_{002}^{\varepsilon, \ell}-2 c_{101}^{\varepsilon, \ell}+c_{200}^{\varepsilon, \ell} .
\end{aligned}
$$

Using the definition of the Bernstein basis polynomials, it is not difficult to see that $\left|d\left(V^{\varepsilon}\right)\right| \leq C_{1}\left\|w-w^{\varepsilon}\right\|^{2}$, where $\|\cdot\|$ denotes the Euclidean distance in $\mathbb{R}^{2}$, and $C_{1}$ is independent of the perturbation. Since $w^{\varepsilon}$ is an analytic function of $V^{\varepsilon}$, we conclude that $d$ is differentiable at $V$ and its differential is zero. Moreover, by the same argument, $d$ is differentiable with zero differential for any sufficiently slightly perturbed $V^{\varepsilon}$ such that $w^{\varepsilon}=u$. If $w^{\varepsilon} \neq u$, then $d$ is differentiable at $V^{\varepsilon}$ by Cases 1 and 2. To see that $d$ is continuously differentiable at $V$, we note that $\tilde{c}_{020}^{\varepsilon, \ell}, \tilde{c}_{011}^{\varepsilon, \ell}, \tilde{c}_{002}^{\varepsilon, \ell}$ are analytic functions of the vertices, and thus possess bounded partial derivatives in the neighborhood of $V$. By investigating the derivatives of $B_{020}^{\varepsilon, \ell}(u), B_{011}^{\varepsilon, 1}(u), B_{002}^{\varepsilon, \ell}(u)$ with respect to the coordinates of the vertices in $V^{\varepsilon}$, we arrive at the upper bound $C_{2} \| w-$ $w^{\varepsilon} \|$ for the absolute values of all partial derivatives of $d\left(V^{\varepsilon}\right)$ with respect to the coordinates of the vertices in $V^{\varepsilon}$, where the constant $C_{2}$ is independent of the perturbation. This upper bound shows that the differential of $d\left(V^{\varepsilon}\right)$ converges to zero as $V^{\varepsilon} \rightarrow V$, confirming the continuous differentiability in this case. 


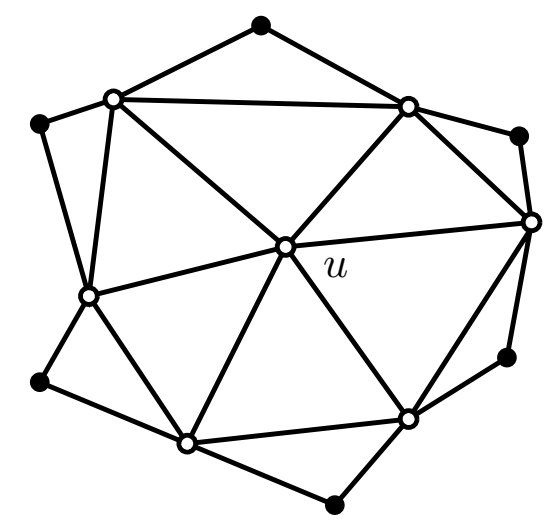

Figure 2: Points in $V_{u}$ (open dots) and $\tilde{V}_{u} \backslash V_{u}$ (black dots).

We have observed above that if $u$ lies in a triangle $T \in \triangle$, then the value $s(V, z, u)$ depends only on the data values at the three vertices of $T$. It also depends on the location of these three vertices, but due to the nature of the Powell-Sabin split, also on the location of the vertices of any triangle in $\triangle$ that shares an edge with $T$. The same holds for the partial derivatives $D_{p} s(V, z, u)$ of $s$ with respect to the parameters $p$ in the sets $V, z$ when $u$ is in the interior of some triangle $T \in \triangle$. However, the above proof shows that when $u$ is on an edge or at a vertex of $T$, some of the $D_{p} s(V, z, u)$ may depend on a larger set of data and vertices. This is due to the fact that if we perturb $\triangle, u$ may fall into a triangle different from the perturbation $T^{\varepsilon}$ of $T$. More precisely, $D_{p} s(V, z, u)$ depends on the location of (and data at) vertices in the set

$$
V_{u}:=\{v \in V: u \in T \text { for a triangle } T \in \triangle \text { with a vertex at } v\} .
$$

But taking account of the fact that the Powell-Sabin split of a given triangle $T$ also depends on the vertices of all triangles that share an edge with $T$, we conclude that $D_{p} s(V, z, u)$ depends on the location of vertices in the set

$$
\tilde{V}_{u}:=\left\{v \in V: \exists \text { a triangle with vertices } v \text { and } v_{1}, v_{2} \in V_{u}\right\} .
$$

Combining these observations shows that $D_{p} s(V, z, u)$ depends on the location of vertices in $\tilde{V}_{u}$ and data at $V_{u}$.

\section{Manifolds: Preliminaries}

\subsection{Atlases}

Let $\Omega$ be a compact 2 -dimensional smooth manifold without boundary. For each $\xi \in \Omega$, suppose that $U_{\xi}$ is an open subset of $\Omega$ containing $\xi$, and that 
$\phi_{\xi}: U_{\xi} \rightarrow \mathbb{R}^{2}$ is a homeomorphism between $U_{\xi}$ and an open subset of $\mathbb{R}^{2}$. Suppose also that for every $\xi, \zeta \in \Omega, \phi_{\zeta} \circ \phi_{\xi}^{-1}: \phi_{\xi}\left(U_{\zeta} \cap U_{\xi}\right) \rightarrow \phi_{\zeta}\left(U_{\zeta} \cap U_{\xi}\right)$ is a $C^{1}$ mapping whenever $U_{\xi} \cap U_{\zeta} \neq \emptyset$. Then, according to standard terminology, see e.g. [19], $\Phi=\left\{\left(U_{\xi}, \phi_{\xi}\right)\right\}_{\xi \in \Omega}$ is an atlas for $\Omega$, and $\left(U_{\xi}, \phi_{\xi}\right), \xi \in \Omega$, are its charts. We emphasize that we need a chart for each $\xi \in \Omega$, rather than simply a covering of $\Omega$ by charts, as is usually required of an atlas. Moreover, we suppose that the charts depend smoothly on $\xi$ in the sense of Definition 3.1 below. Let

$$
B_{\xi}:=\phi_{\xi}\left(U_{\xi}\right), \quad \phi_{\zeta \xi}:=\phi_{\zeta} \circ \phi_{\xi}^{-1} .
$$

Then $B_{\xi}$ is an open set in $\mathbb{R}^{2}$, and $\phi_{\zeta \xi}$ is an invertible $C^{1}$ mapping between $\phi_{\xi}\left(U_{\zeta} \cap U_{\xi}\right) \subset B_{\xi}$ and $\phi_{\zeta}\left(U_{\zeta} \cap U_{\xi}\right) \subset B_{\zeta}$.

A real function $f$ defined in a neighborhood of a point $\xi \in \Omega$ is said to be continuous $\left(C^{0}\right)$ at $\xi$ if one of its local representations $f \circ \phi_{\zeta}^{-1}$ is continuous at $\phi_{\zeta}(\xi)$ for some $\zeta$ with $\xi \in U_{\zeta}$. Similarly, we say that $f$ is continuously differentiable $\left(C^{1}\right)$ at $\xi$ provided one of its local representations $f \circ \phi_{\zeta}^{-1}$ is continuously differentiable at $\phi_{\zeta}(\xi)$ for some $\zeta$ with $\xi \in U_{\zeta}$. Since all $\phi_{\zeta \xi}$ are invertible $C^{1}$ mappings, every local representation $f \circ \phi_{\zeta}^{-1}$ will then be $C^{0}$ (resp. $C^{1}$ ) at $\phi_{\zeta}(\xi)$.

For a $C^{1}$ function $f$ defined in a neighborhood $U$ of $\zeta \in \Omega$, we also define $J_{\zeta}(f): U \cap U_{\zeta} \rightarrow \mathbb{R}^{2 \times 2}$ by

$$
J_{\zeta}(f)(\mu):=J\left(f \circ \phi_{\zeta}^{-1}\right)\left(\phi_{\zeta}(\mu)\right), \quad \mu \in U \cap U_{\zeta},
$$

where for any smooth function $g: \mathbb{R}^{2} \rightarrow \mathbb{R}^{2}, g=\left(g^{[1]}, g^{[2]}\right)^{T}, J(g)$ denotes its Jacobian

$$
J(g):=\left[\begin{array}{ll}
\frac{\partial g^{[1]}}{\partial x^{[1]}} & \frac{\partial g^{[1]}}{\partial x^{[2]}} \\
\frac{\partial g^{[2]}}{\partial x^{[1]}} & \frac{\partial g^{[2]}}{\partial x^{[2]}}
\end{array}\right] .
$$

We write

$$
J_{\zeta \xi}:=J_{\xi}\left(\phi_{\zeta}\right), \quad \text { on } U_{\zeta} \cap U_{\xi},
$$

so that

$$
J_{\zeta \xi}(\mu)=J_{\xi}\left(\phi_{\zeta}\right)(\mu)=J\left(\phi_{\zeta \xi}\right)\left(\phi_{\xi}(\mu)\right), \quad \mu \in U_{\zeta} \cap U_{\xi},
$$

is the Jacobian of $\phi_{\zeta \xi}$ evaluated at $\phi_{\xi}(\mu)$. Since $\phi_{\zeta \xi}^{-1}=\phi_{\xi \zeta}$, the well-known properties of the Jacobian imply

$$
\left[J_{\zeta \xi}(\mu)\right]^{-1}=J_{\xi \zeta}(\mu) .
$$


Definition 3.1. We say that the charts $\left(U_{\xi}, \phi_{\xi}\right)$ depend smoothly on $\xi$ if $\phi_{\xi}(\xi)$ is a $C^{1}$ function of $\xi$, and for each $\xi \in \Omega$ there is an open neighborhood $\tilde{U}_{\xi}$ of $\xi$ such that the following conditions hold:

- $\tilde{U}_{\xi} \subset U_{\zeta}$ for all $\zeta$ sufficiently close to $\xi$,

- for any $\zeta$ sufficiently close to $\xi$, there is a rotation or rotoinversion (a rotation followed by a flip) $r_{\zeta}: \mathbb{R}^{2} \rightarrow \mathbb{R}^{2}$ about $\phi_{\zeta}(\zeta)$ such that for any $\mu \in \tilde{U}_{\xi}$, both $\left(r_{\zeta} \circ \phi_{\zeta}\right)(\mu)$ and $J_{\mu}\left(r_{\zeta} \circ \phi_{\zeta}\right)(\mu)$ are $C^{1}$ functions of $\zeta$ at $\zeta=\xi$.

If the charts of a $C^{1}$ atlas $\Phi=\left\{\left(U_{\xi}, \phi_{\xi}\right)\right\}_{\xi \in \Omega}$ depend smoothly on $\xi$, then $\Phi$ is said to be admissible. Any system of neighborhoods $\left\{\tilde{U}_{\xi}\right\}_{\xi \in \Omega}$ satisfying the above conditions is called a basic covering.

It is shown in Section 7 that if $\Omega$ is a 2 -dimensional manifold embedded in $\mathbb{R}^{3}$, then an admissible atlas can be defined using local orthogonal projections onto tangent planes. More specific examples of admissible atlases are given in Remarks 8.12 and 8.13 for the sphere and the torus.

Note that removing local transformations $r_{\zeta}$ from Definition 3.1 would result in severe restrictions on the topology of the manifold $\Omega$. This is related to the famous 'hairy ball' theorem, see Remark 8.7.

\subsection{Gradients}

Suppose that $f$ is a continuously differentiable real-valued function on $\Omega$. For any $\xi \in \Omega$, we write $f_{\xi}:=f \circ \phi_{\xi}^{-1}$. Then $f_{\xi}: B_{\xi} \rightarrow \mathbb{R}$ is a bivariate $C^{1}$ function. For any $\mu \in U_{\xi}$, we write

$$
\nabla_{\xi} f(\mu):=\nabla f_{\xi}\left(\phi_{\xi}(\mu)\right)
$$

for the value of the gradient $\nabla f_{\xi}:=\left(\frac{\partial f_{\xi}}{\partial x^{(1)}}, \frac{\partial f_{\xi}}{\partial x^{(2)}}\right)$ of $f_{\xi}$ at $\phi_{\xi}(\mu)$. If $U_{\zeta} \cap U_{\xi} \neq \emptyset$, then $f_{\xi}=f_{\zeta} \circ \phi_{\zeta \xi}$ on $\phi_{\xi}\left(U_{\zeta} \cap U_{\xi}\right)$, and by the chain rule,

$$
\nabla_{\xi} f(\mu)=\nabla_{\zeta} f(\mu) J_{\zeta \xi}(\mu), \quad \mu \in U_{\xi} \cap U_{\zeta} .
$$

\subsection{Sobolev spaces}

Given a continuous function $f: \Omega \rightarrow \mathbb{R}$, we define its maximum norm to be $\|f\|_{C(\Omega)}:=\max _{\xi \in \Omega}|f(\xi)|$. Given $r \geq 1$, we say that $f$ belongs to the Sobolev 
space $W_{\infty}^{r}(\Omega)$ provided $f_{\xi} \in W_{\infty}^{r}\left(B_{\xi}\right)$ for all $\xi \in \Omega$. We define the Sobolev norm on $W_{\infty}^{r}(\Omega)$ by

$$
\|f\|_{W_{\infty}^{r}(\Omega)}:=\max _{\xi \in \Omega}\left\|f_{\xi}\right\|_{W_{\infty}^{r}\left(B_{\xi}\right)} .
$$

This definition of the Sobolev norm for the functions defined on the manifold $\Omega$ is equivalent to the standard definitions, see [13].

\subsection{Manifold Triangulations}

Given a finite set $\mathcal{V}$ of points in a manifold $\Omega$, let $\mathcal{T}$ be a set of triples $\tau=\{v, u, w\}$ of points $v, u, w \in \mathcal{V}$ such that

- any two triples have at most two common points,

- any pair of points in $\mathcal{V}$ belong to at most two different triples in $\mathcal{T}$,

- for any $v \in \mathcal{V}$, the set of all triples containing $v$ forms a cell, i.e. $\{\tau \in \mathcal{T}: v \in \tau\}=\left\{\tau_{i}\right\}_{i=1}^{n}$ for some $n \geq 3$, where $\tau_{i}=\left\{v, v_{i}, v_{i+1}\right\}$, with distinct $v_{1}, \ldots, v_{n}$, and $v_{n+1}=v_{1}$.

If these conditions are satisfied, we say that $\mathcal{T}$ is a triangulation of $\Omega$ with vertices $\mathcal{V}$. We say that two vertices $v_{1}, v_{2}$ are connected in $\mathcal{T}$ if there is a triple $\tau \in \mathcal{T}$ containing both $v_{1}$ and $v_{2}$. This definition of a triangulation of a manifold $\Omega$ is described by connectivity of vertices only, and does not involve "edges" or "triangles" on $\Omega$. Indeed, $\mathcal{T}$ is essentially an abstract simplicial complex [25] with vertices in $\Omega$.

It is well known that any compact 2 -dimensional manifold $\Omega$ can be triangulated (see $[20,24]$ ), i.e. there exists a finite triangulation $\mathcal{T}$ of $\Omega$ in the above sense along with a corresponding partition of $\Omega$ into homeomorphic images of triangles, similar to a planar triangulation. Such a partition is also called a triangulation of $\Omega$, but since we never make use of it in this paper, there will be no confusion with our definition.

We now assume that $\Phi=\left\{\left(U_{\xi}, \phi_{\xi}\right)\right\}_{\xi \in \Omega}$ is an admissible atlas for $\Omega$ in the sense of Definition 3.1. Let $\xi \in \Omega$, and suppose that all vertices of $\tau=\{v, u, w\} \in \mathcal{T}$ are in $U_{\xi}$. Then we denote by $\phi_{\xi}(\tau)$ the planar triangle with vertices $\phi_{\xi}(u), \phi_{\xi}(v), \phi_{\xi}(w)$. Note that the triangle $\phi_{\xi}(\tau)$ may be degenerate.

Definition 3.2. We say that a triangulation $\mathcal{T}$ of $\Omega$ is consistent with $\Phi$ provided that there is a basic covering $\left\{\tilde{U}_{\xi}\right\}_{\xi \in \Omega}$ such that for any $\xi \in \Omega$, 
- $\triangle_{\xi}:=\left\{\phi_{\xi}(\tau): \tau \in \mathcal{T}, \tau \subset \tilde{U}_{\xi}\right\}$ is a planar triangulation of $P_{\xi}:=$ $\cup_{T \in \triangle_{\xi}} T$ (in particular, every triangle $T \in \triangle_{\xi}$ is non-degenerate),

- $\phi_{\xi}(\xi)$ lies in the interior of $P_{\xi}$.

For any $\xi \in \Omega$, let $\mathcal{V}_{\xi}$ be the set consisting of vertices of all $\tau \in \mathcal{T}_{\xi}:=$ $\left\{\tau \in \mathcal{T}: \tau \subset \tilde{U}_{\xi}\right\}$ such that $\phi_{\xi}(\xi)$ lies in $\phi_{\xi}(\tau)$, i.e.

$$
\mathcal{V}_{\xi}:=\left\{v \in \mathcal{V} \cap U_{\xi}: \phi_{\xi}(\xi) \in \phi_{\xi}(\tau) \text { for a } \tau \in \mathcal{T}_{\xi} \text { with a vertex at } v\right\} \text {. }
$$

For a consistent triangulation $\mathcal{T}, \mathcal{V}_{\xi}$ is independent of the basic covering choosen. If $\xi$ is a vertex in $\mathcal{V}$, then $\mathcal{V}_{\xi}$ consists of $\xi$ and all vertices connected to it. For any point $\xi \in \Omega \backslash \mathcal{V}$ the set $\mathcal{V}_{\xi}$ contains either three or four points, depending on whether $\phi_{\xi}(\xi)$ belongs to the interior of a triangle in $\triangle_{\xi}$, or lies on a common edge of two such triangles. We say that $\xi$ is an interior point or respectively an edge point with respect to the triangulation $\mathcal{T}$. Clearly, for any basic covering,

$$
\mathcal{V}_{\xi} \subset \tilde{U}_{\xi} \quad \text { and } \quad \phi_{\xi}\left(\mathcal{V}_{\xi}\right) \subset P_{\xi}
$$

Lemma 3.3. Let $\mathcal{T}$ be a triangulation consistent with an admissible atlas $\Phi$ for $\Omega$. Then for any $\xi \in \Omega, \mathcal{V}_{\zeta} \subseteq \mathcal{V}_{\xi}$ for all $\zeta \in \Omega$ sufficiently close to $\xi$.

Proof. Let $\mathcal{V}_{\xi}=\left\{v_{1}, \ldots, v_{m}\right\}$. Since $\mathcal{V}_{\xi} \subset \tilde{U}_{\xi}$, the functions $v_{i}(\zeta):=\left(r_{\zeta} \circ\right.$ $\left.\phi_{\zeta}\right)\left(v_{i}\right), i=1, \ldots, m$, are well defined for all $\zeta$ sufficiently close to $\xi$, and continuous at $\zeta=\xi$, c.f. Definition 3.1. Also $\left(r_{\zeta} \circ \phi_{\zeta}\right)(\zeta)=\phi_{\zeta}(\zeta)$ is a continuous function of $\zeta$. Denote by $\Lambda_{\zeta}$ the union of all triangles of the type $r_{\zeta}\left(\phi_{\zeta}(\tau)\right), \tau \in \mathcal{T}$, whose vertices are all in $\left(r_{\zeta} \circ \phi_{\zeta}\right)\left(\mathcal{V}_{\xi}\right)=\left\{v_{1}(\zeta), \ldots, v_{m}(\zeta)\right\}$. Clearly, $\phi_{\xi}(\xi)$ lies in the interior of $\Lambda_{\xi}$. By the above continuity we conclude that for all $\zeta$ sufficiently close to $\xi, \phi_{\zeta}(\zeta)$ lies in the interior of $\Lambda_{\zeta}$. Hence $\phi_{\zeta}(\zeta) \in \phi_{\zeta}(\tau)$ implies that all vertices of $\tau$ are in $\mathcal{V}_{\xi}$, and $\mathcal{V}_{\zeta} \subseteq \mathcal{V}_{\xi}$ follows.

It is easy to see that the set of all interior points with respect to a consistent triangulation is an open subset of $\Omega$. Indeed, if $\xi$ is an interior point, then $\mathcal{V}_{\xi}$ consists of just three vertices. For any $\zeta$ sufficiently close to $\xi$, $\mathcal{V}_{\zeta} \subset \mathcal{V}_{\xi}$ and $\mathcal{V}_{\zeta}$ cannot have fewer than three vertices. Hence $\mathcal{V}_{\zeta}=\mathcal{V}_{\xi}$ and $\zeta$ is an interior point. In addition to consistency, we will need the following assumption specifically related to the Powell-Sabin spline:

for every $\xi \in \Omega$, if $\phi_{\xi}(\xi) \in T$ for a $T \in \triangle_{\xi}$, then $\triangle_{\xi}$ also includes three triangles sharing edges with $T$.

We extend $\mathcal{V}_{\xi}$ to $\tilde{\mathcal{V}}_{\xi}$ by adding to $\mathcal{V}_{\xi}$ the vertices of the triangles described in (3.4). The definition of $\triangle_{\xi}$ implies that $\tilde{\mathcal{V}}_{\xi} \subset \tilde{U}_{\xi}$. 


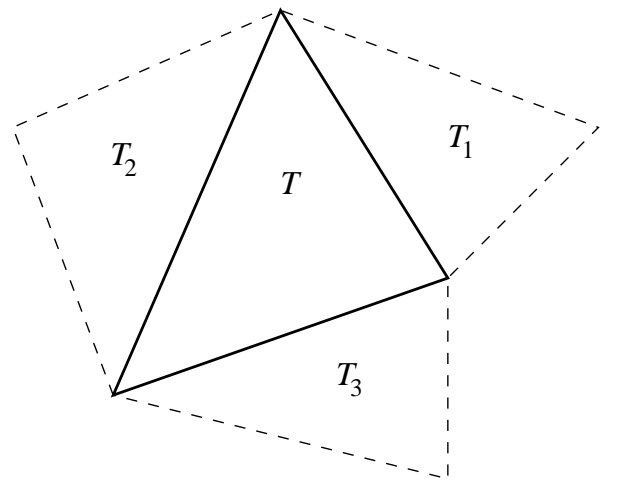

(a)

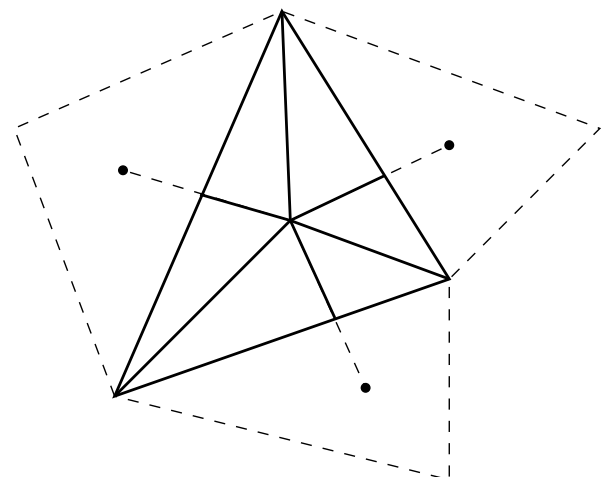

(b)

Figure 3: Powell-Sabin split of a triangle.

\section{An Interpolation Method}

Let $\mathcal{T}$ be a triangulation of $\Omega$ consistent with an admissible atlas $\Phi=$ $\left\{\left(U_{\xi}, \phi_{\xi}\right)\right\}_{\xi \in \Omega}$. We assume that $\mathcal{T}$ is fine enough for (3.4) to hold. Let $D:=\left\{a_{v}, \sigma_{v}\right\}_{v \in \mathcal{V}}$, where $a_{v}$ are real numbers and $\sigma_{v}=\left(\sigma_{v}^{[1]}, \sigma_{v}^{[2]}\right)$ are 2vectors. We now show how to construct a $C^{1}$ function $s_{\mathcal{T}}$ defined on $\Omega$ that satisfies the interpolation conditions

$$
s_{\mathcal{T}}(v)=a_{v}, \quad \nabla_{v} s_{\mathcal{T}}(v)=\sigma_{v}, \quad \text { all } v \in \mathcal{V} .
$$

Algorithm 4.1. Given $\xi \in \Omega$, compute $s_{\mathcal{T}}(\xi)$ :

1. Let $T:=\left\langle w_{1}, w_{2}, w_{3}\right\rangle$ be a triangle in $\triangle_{\xi}$ such that $\phi_{\xi}(\xi) \in T$, and let $T_{1}:=\left\langle w_{4}, w_{3}, w_{2}\right\rangle, T_{2}:=\left\langle w_{5}, w_{1}, w_{3}\right\rangle$, and $T_{3}:=\left\langle w_{6}, w_{2}, w_{1}\right\rangle$ be the three triangles in $\triangle_{\xi}$ sharing edges with $T$, see Figure 3(a).

2. Let $T_{P S}$ be the Powell-Sabin split of $T$ into six triangles obtained by connecting the incenter $w$ of $T$ to the incenters of $T_{1}, T_{2}, T_{3}$, and to the vertices $w_{1}, w_{2}, w_{3}$, see Figure $3(b)$.

3. Let $g_{i}:=\sigma_{v_{i}} J_{v_{i} \xi}\left(v_{i}\right)$, where $v_{i}=\phi_{\xi}^{-1}\left(w_{i}\right)$, for $i=1,2,3$.

4. Let $s_{\mathcal{T}}(\xi)$ be the value at $\phi_{\xi}(\xi)$ of the Powell-Sabin $C^{1}$ quadratic spline $s_{\xi}$ defined on $T_{P S}$ that satisfies $s_{\xi}\left(w_{i}\right)=a_{v_{i}}$ and $\nabla s_{\xi}\left(w_{i}\right)=g_{i}$ for $i=1,2,3$. 
Since the Powell-Sabin interpolant in Step 4) is uniquely defined by the values $\left\{\left(a_{v_{i}}, g_{i}\right)\right\}_{i=1}^{3}$ at the vertices $\left\{w_{i}\right\}_{i=1}^{3}$, it follows that $s_{\mathcal{T}}$ is uniquely defined by the data $D$. By construction, $s_{\mathcal{T}}$ satisfies (4.1).

Theorem 4.2. The interpolant $s_{\mathcal{T}}$ is a $C^{1}$ function on the manifold $\Omega$.

Proof. We may assume without loss of generality that for any $\zeta \in \Omega$ the point $\phi_{\zeta}(\zeta)$ is the origin in $\mathbb{R}^{2}$, since otherwise we may replace $\phi_{\zeta}$ by $\phi_{\zeta}-$ $\phi_{\zeta}(\zeta)$, and $s_{\zeta}$ by $s_{\zeta}\left(\cdot+\phi_{\zeta}(\zeta)\right)$, which coincides with the Powell-Sabin spline computed with respect to the shifted version of the local triangulation $\triangle_{\zeta}$.

Fix $\xi \in \Omega$. Since we are assuming that the charts of $\Phi$ depend smoothly on $\xi$, it follows that for any $\zeta$ sufficiently close to $\xi$ there is a rotation or rotoinversion $r_{\zeta}: \mathbb{R}^{2} \rightarrow \mathbb{R}^{2}$ about the origin (an orthogonal linear transformation of the plane) such that both $\left(r_{\zeta} \circ \phi_{\zeta}\right)(\mu)$ and $J_{\mu}\left(r_{\zeta} \circ \phi_{\zeta}\right)(\mu)$, as functions of $\zeta$, are continuously differentiable at $\zeta=\xi$ as soon as $\mu \in \tilde{U}_{\xi}$. Without loss of generality we assume that $r_{\xi}$ is the identity. Recall from (3.3) that $\mathcal{V}_{\xi}$ denotes the set of all vertices $v \in \mathcal{V} \cap U_{\xi}$ such that $\phi_{\xi}(\xi) \in T$ for a triangle $T \in \triangle_{\xi}$ attached to $\phi_{\xi}(v)$. Since $\mathcal{T}$ is consistent with the atlas $\Phi$, by Lemma 3.3 we may choose a neighborhood $U \subset U_{\xi}$ of $\xi$ such that $\mathcal{V}_{\zeta} \subseteq \mathcal{V}_{\xi} \subset \tilde{U}_{\xi}$ for all $\zeta \in U$. Moreover, according to Definition 3.1, we may choose a smaller $U$ to ensure that $\tilde{U}_{\xi} \subset U_{\zeta}$ for all $\zeta \in U$. For any $\zeta \in U$ it follows by Definition 3.2 that all points in $\phi_{\zeta}\left(\mathcal{V}_{\zeta}\right)$ are vertices of $\triangle_{\zeta}$. Clearly, $\triangle_{\zeta}$ includes all triangles $\phi_{\zeta}(\tau)$ for $\tau \in \mathcal{T}$ with vertices in $\mathcal{V}_{\zeta}$. In view of (3.4), it also includes images of all $\tau \in \mathcal{T}$ having a pair of vertices in $\mathcal{V}_{\zeta}$. Moreover, $\tilde{\mathcal{V}}_{\xi} \subset \tilde{U}_{\xi} \subset U_{\zeta}$.

Now for any $\zeta \in U$ and each vertex $v \in \tilde{\mathcal{V}}_{\xi}$, set $v(\zeta)=\left(r_{\zeta} \circ \phi_{\zeta}\right)(v)$. The functions $v(\zeta)$ are continuously differentiable at $\zeta=\xi$. Let $\tilde{\triangle}_{\zeta}$ denote the triangulation obtained by applying $r_{\zeta}$ to $\triangle_{\zeta}$, and let $\tilde{\triangle}_{\zeta, P S}$ be the PowellSabin split of $\tilde{\triangle}_{\zeta}$. Since $r_{\zeta}$ is an orthogonal transformation, it is easy to see that $s_{\mathcal{T}}(\zeta)$ can be computed as the value at the origin of the PowellSabin spline $\tilde{s}_{\zeta}$ defined on $\tilde{\triangle}_{\zeta, P S}$ that interpolates the values $\left\{\left(a_{v}, g(\zeta)\right)\right\}$ at the vertices $v(\zeta)$ for all $v \in \tilde{\mathcal{V}}_{\xi}$, where $g(\zeta)=\sigma_{v} J_{v \zeta}(v) J\left(r_{\zeta}^{-1}\right)$. Since $J_{v \zeta}(v) J\left(r_{\zeta}^{-1}\right)=\left[J_{v}\left(r_{\zeta} \circ \phi_{\zeta}\right)(v)\right]^{-1}$ is continuously differentiable with respect to $\zeta$ at $\zeta=\xi$, and since by Theorem 2.1 the value of the Powell-Sabin interpolant $s_{\zeta}$ at the origin depends smoothly on the vertex locations and the data, we conclude that $s_{\mathcal{T}}$ is continuously differentiable at $\xi$.

Suppose that $s_{\mathcal{T}}(f)$ is the interpolant corresponding to the data

$$
a_{v}:=f(v), \quad \sigma_{v}:=\nabla_{v} f(v), \quad \text { all } v \in \mathcal{V},
$$


where $f$ is a smooth function defined on $\Omega$. We now show that $s_{\mathcal{T}}(f)$ approximates $f$ to order $\mathcal{O}\left(h^{3}\right)$, where $h$ is the mesh size of $\mathcal{T}$, i.e., the length of the longest edge in the triangles in the set $\left\{\triangle_{\xi}\right\}_{\xi \in \Omega}$. Let $\alpha$ be the smallest angle appearing in the triangles in this set.

Theorem 4.3. Let $f \in W_{\infty}^{3}(\Omega)$. Then

$$
\left\|f-s_{\mathcal{T}}(f)\right\|_{C(\Omega)} \leq K h^{3}\|f\|_{W_{\infty}^{3}(\Omega)},
$$

where $K$ is a constant depending only on $\alpha$.

Proof. Fix $\xi \in \Omega$, and let $s_{\xi}$ be the bivariate Powell-Sabin spline defined on the triangulation $\triangle_{\xi}$ that interpolates the values $\left\{\left(a_{v_{i}}, g_{i}\right)\right\}_{i=1}^{3}$ at the vertices $\left\{w_{i}\right\}_{i=1}^{3}$ of the triangle in $\triangle_{\xi}$ containing $\xi$ as described in Algorithm 4.1. Then

$$
f_{\xi}\left(w_{i}\right)=f\left(v_{i}\right)=a_{v_{i}}=s_{\xi}\left(w_{i}\right), \quad i=1,2,3,
$$

and by (3.2)

$$
\nabla f_{\xi}\left(w_{i}\right)=\nabla_{\xi} f\left(v_{i}\right)=\nabla_{v_{i}} f\left(v_{i}\right) J_{v_{i} \xi}\left(v_{i}\right)=\sigma_{v_{i}} J_{v_{i} \xi}\left(v_{i}\right)=\nabla s_{\xi}\left(w_{i}\right),
$$

$i=1,2,3$.

Thus, $s_{\xi}$ interpolates the function values and gradients of $f_{\xi}$ at $w_{1}, w_{2}, w_{3}$. It follows from well-known error bounds for bivariate Powell-Sabin interpolation [30] (see also [21]) that

$$
\left|f_{\xi}(\xi)-s_{\xi}(\xi)\right| \leq K_{1} h^{3}\left\|f_{\xi}\right\|_{W_{\infty}^{3}\left(B_{\xi}\right)},
$$

where $K_{1}$ is a constant depending only on the smallest angle in $\triangle_{\xi}$. By the definition of the Sobolev norm on $\Omega$, taking the maximium over $\xi \in \Omega$ gives (4.2).

\section{A Two-Stage Data Fitting Method}

In practice we are frequently given only values of an unknown function $f$ at a set $X$ of scattered data points on the manifold $\Omega$. In this case we can use a two-stage method to construct an approximation. First we select a consistent triangulation $\mathcal{T}$ of $\Omega$ satisfying (3.4). Let $\mathcal{V}$ be the set of vertices of $\mathcal{T}$. Note that we do not require that the vertices be located at the data 
points of $X$, and the number of vertices may be different (in particular much smaller) than the number of data points.

In the first stage of the algorithm we compute local approximations to the values $\left\{f(v), \nabla_{v} f(v)\right\}_{v \in \mathcal{V}}$ based on the data $\{f(\xi)\}_{\xi \in X}$. We perform these calculations in the sets $B_{v} \subset \mathbb{R}^{2}$ using techniques available for local fitting of bivariate data. To carry this out, we suppose that $X$ is sufficiently dense to satisfy

$$
X \cap U_{v} \neq \emptyset \text { for each } v \in \mathcal{V} .
$$

Clearly, (5.1) is just a minimum requirement needed to formulate the algorithm. It does not guarantee that accurate local approximations based on the data in $X \cap U_{v}$ can be computed.

Experience with the bivariate case [10] suggests that for each $v \in \mathcal{V}$, we compute both $a_{v} \approx f(v)$ and $\sigma_{v} \approx \nabla_{v} f(v)$ by averaging several estimates of the same quantities based on different sets of nearby data. It follows from the consistency of $\mathcal{T}$ that for each vertex $v \in \mathcal{V}$, all vertices of $\mathcal{T}$ connected to $v$ belong to the set $U_{v}$.

Algorithm 5.1. Given $\{f(\xi)\}_{\xi \in X}$, compute $\left\{a_{v}, \sigma_{v}\right\}_{v \in \mathcal{V}}$ as follows:

1. For each $v \in \mathcal{V}$ :

(a) Let $v_{0}:=v$, and let $v_{1}, v_{2}, \ldots, v_{n} \in \mathcal{V}$ be the set of vertices of $\mathcal{T}$ connected to $v$. Let $\tilde{v}_{i}=\phi_{v}\left(v_{i}\right), i=1, \ldots, n$.

(b) Choose a set $\tilde{X}_{v} \subset \phi_{v}\left(X \cap U_{v}\right)$ of points in $B_{v}$ near $\phi_{v}(v)$.

(c) Compute a bivariate approximation $p_{v}$ defined on $B_{v}$ based on the $\operatorname{data}\left\{f_{v}(\tilde{\xi})\right\}_{\tilde{\xi} \in \tilde{X}_{v}}$, where $f_{v}:=f \circ \phi_{v}^{-1}$.

(d) Store the numbers $a_{v, v_{i}}:=p_{v}\left(\tilde{v}_{i}\right)$ and vectors $\sigma_{v, v_{i}}:=\nabla p_{v}\left(\tilde{v}_{i}\right) J_{v v_{i}}\left(v_{i}\right)$ for $i=0, \ldots, n$.

2. For each $v \in \mathcal{V}$, set

$$
a_{v}:=\frac{1}{n+1} \sum_{i=0}^{n} a_{v_{i}, v}, \quad \sigma_{v}:=\frac{1}{n+1} \sum_{i=0}^{n} \sigma_{v_{i}, v} .
$$

In the second stage of the algorithm we construct our approximant $s_{\mathcal{T}}$ as the interpolant (4.1) to the data $\left\{a_{v}, \sigma_{v}\right\}_{v \in \mathcal{V}}$ obtained from Algorithm 5.1.

We have not specified how $\mathcal{T}$ is selected and how the Steps 1(b) and 1(c) of Algorithm 5.1 are to be performed. However, the overall performance of 
the two-stage method will depend significantly on the particular techniques used in these steps. Numerical examples in our paper [9] make use of recently developed adaptive techniques based on local least squares fitting by bivariate polynomials and radial basis functions $[7,8,10]$.

\section{An Error Bound for the Two-Stage Method}

Suppose that $f$ is a smooth function defined on $\Omega$, and that $s_{\mathcal{T}}=s_{\mathcal{T}}(f)$ is the approximant of $f$ constructed in the previous section based on values $\{f(\xi)\}_{\xi \in X}$ of $f$ at some scattered set $X$ of data points on $\Omega$. Let $h$ be the mesh size of the triangulation $\mathcal{T}$. In this section we show that $s_{\mathcal{T}}$ approximates $f$ to order $h^{3}$ as $h \rightarrow 0$, provided that for each vertex $v$ of $\mathcal{T}$, the local approximation $p_{v}$ of $f_{v}:=f \circ \phi_{v}^{-1}$ approximates the function value $f_{v}(v)$ to order at least $h^{3}$, and the first derivatives of $f_{v}$ at $v$ to order $h^{2}$.

In this section we denote by $\|\cdot\|_{2}$ the Euclidean vector norm as well as the corresponding matrix norm. For a vector function $g: G \rightarrow \mathbb{R}^{2}$, we set $\|g\|_{C(G)}:=\max _{x \in G}\|g(x)\|_{2}$.

Given $\xi \in \Omega$, let $\triangle_{\xi}$ be the associated planar triangulation, as in Section 3.4. Let

$$
\kappa(\xi):=\max _{v \in \mathcal{V}_{\xi}}\left\{\left\|J_{\xi v}(v)\right\|_{2},\left\|J_{v \xi}(v)\right\|_{2}\right\},
$$

where $\mathcal{V}_{\xi}$ is defined in (3.3). We assume that

$$
\kappa:=\sup _{\xi \in \Omega} \kappa(\xi)<\infty .
$$

For each $v \in \mathcal{V}$, let $N_{v}$ be the union of all triangles of $\triangle_{v}$ attached to $v$, and let $p_{v}$ be the bivariate approximation to $f_{v}$, as in Algorithm 5.1. Recall that $B_{v}:=\phi_{v}\left(U_{v}\right)$.

Theorem 6.1. Let $f \in W_{\infty}^{3}(\Omega)$. Then

$$
\begin{aligned}
\left\|f-s_{\mathcal{T}}\right\|_{C(\Omega)} & \leq K\left[h^{3}\|f\|_{W_{\infty}^{3}(\Omega)}\right. \\
& \left.+\max _{v \in \mathcal{V}}\left\{\left\|f_{v}-p_{v}\right\|_{C\left(N_{v} \cap B_{v}\right)}+h\left\|\nabla f_{v}-\nabla p_{v}\right\|_{C\left(N_{v} \cap B_{v}\right)}\right\}\right],
\end{aligned}
$$

where $K$ is a constant depending only on $\kappa$ and the smallest angle $\alpha$. 
Proof. Let $\xi \in \Omega$. We have $\left|f(\xi)-s_{\mathcal{T}}(\xi)\right|=\left|f_{\xi}(\xi)-s_{\xi}(\xi)\right|$, where $f_{\xi}:=f \circ \phi_{\xi}^{-1}$ and $s_{\xi}$ is the bivariate Powell-Sabin interpolating spline on the planar triangulation $\triangle_{\xi}$, computed using the values $a_{v_{i}}$ and vectors $\sigma_{v_{i}}$ corresponding to the vertices $\left\{w_{i}:=\phi_{\xi}\left(v_{i}\right)\right\}_{i=1}^{3}$ of the triangle $T$ in $B_{\xi}$ that contains $\phi_{\xi}(\xi)$. Recall that $a_{v_{i}}, \sigma_{v_{i}}$ are computed in the first stage using Algorithm 5.1. By Algorithm 4.1,

$$
s_{\xi}\left(w_{i}\right)=a_{v_{i}}, \quad \nabla s_{\xi}\left(w_{i}\right)=\sigma_{v_{i}} J_{v_{i} \xi}\left(v_{i}\right), \quad i=1,2,3 .
$$

Let $\hat{s}$ be the bivariate Powell-Sabin spline on the triangulation $\triangle_{\xi}$ interpolating the exact values and gradients of $f_{\xi}$, i.e.,

$$
\hat{s}\left(w_{i}\right)=\hat{a}_{v_{i}}:=f_{\xi}\left(w_{i}\right)=f\left(v_{i}\right), \quad \nabla \hat{s}\left(w_{i}\right)=\hat{\sigma}_{v_{i}}:=\nabla f_{\xi}\left(w_{i}\right)=\nabla_{\xi} f\left(v_{i}\right),
$$

for $i=1,2,3$. Then by Theorem 4.3,

$$
\left|f_{\xi}(\xi)-\hat{s}(\xi)\right| \leq K_{1} h^{3}\|f\|_{W_{\infty}^{3}(\Omega)},
$$

where $K_{1}$ is a constant depending only on $\alpha$. Now by a standard argument involving the cardinal functions of Powell-Sabin interpolation,

$$
\left|\hat{s}(\xi)-s_{\xi}(\xi)\right| \leq K_{2} \max _{i=1,2,3}\left\{\left|\hat{a}_{v_{i}}-a_{v_{i}}\right|+h\left\|\hat{\sigma}_{v_{i}}-\sigma_{v_{i}} J_{v_{i} \xi}\left(v_{i}\right)\right\|_{2}\right\},
$$

with a constant $K_{2}$ depending only on $\alpha$. For each $i=1,2,3$,

$$
\hat{a}_{v_{i}}-a_{v_{i}}=f\left(v_{i}\right)-\frac{1}{n+1} \sum_{j=0}^{n} a_{u_{j}, v_{i}}=\frac{1}{n+1} \sum_{j=0}^{n}\left[f_{u_{j}}\left(w_{i j}\right)-p_{u_{j}}\left(w_{i j}\right)\right],
$$

where $u_{0}=v_{i}$, the $u_{1}, \ldots, u_{n}$ are the vertices of $\mathcal{T}$ connected to $v_{i}$, and $w_{i j}=\phi_{u_{j}}\left(v_{i}\right)$. Since $w_{i j} \in N_{u_{j}} \cap B_{u_{j}}$, it follows that

$$
\left|f_{u_{j}}\left(w_{i j}\right)-p_{u_{j}}\left(w_{i j}\right)\right| \leq\left\|f_{u_{j}}-p_{u_{j}}\right\|_{C\left(N_{u_{j}} \cap B_{u_{j}}\right)}
$$

and hence

$$
\left|\hat{a}_{v_{i}}-a_{v_{i}}\right| \leq \max _{v \in \mathcal{V}}\left\|f_{v}-p_{v}\right\|_{C\left(N_{v} \cap B_{v}\right)}, \quad i=1,2,3 .
$$

Similarly, by (3.2) and the definition of $\sigma_{v_{i}}$,

$$
\begin{gathered}
\hat{\sigma}_{v_{i}}-\sigma_{v_{i}} J_{v_{i} \xi}\left(v_{i}\right)=\nabla_{\xi} f\left(v_{i}\right)-\frac{1}{n+1} \sum_{j=0}^{n} \sigma_{u_{j}, v_{i}} J_{v_{i} \xi}\left(v_{i}\right) \\
=\frac{1}{n+1} \sum_{j=0}^{n}\left(\nabla_{v_{i}} f\left(v_{i}\right)-\sigma_{u_{j}, v_{i}}\right) J_{v_{i} \xi}\left(v_{i}\right) .
\end{gathered}
$$


Since $\sigma_{u_{j}, v_{i}}=\nabla p_{u_{j}}\left(w_{i j}\right) J_{u_{j} v_{i}}\left(v_{i}\right)$ and $\nabla_{u_{j}} f\left(v_{i}\right)=\nabla f_{u_{j}}\left(w_{i j}\right)$, (3.2) implies

$$
\begin{aligned}
\nabla_{v_{i}} f\left(v_{i}\right)-\sigma_{u_{j}, v_{i}} & =\left(\nabla_{u_{j}} f\left(v_{i}\right)-\nabla p_{u_{j}}\left(w_{i j}\right)\right) J_{u_{j} v_{i}}\left(v_{i}\right) \\
& =\left(\nabla f_{u_{j}}\left(w_{i j}\right)-\nabla p_{u_{j}}\left(w_{i j}\right)\right) J_{u_{j} v_{i}}\left(v_{i}\right)
\end{aligned}
$$

Hence

$$
\begin{aligned}
\left\|\nabla_{v_{i}} f\left(v_{i}\right)-\sigma_{u_{j}, v_{i}}\right\|_{2} & \leq\left\|\nabla f_{u_{j}}\left(w_{i j}\right)-\nabla p_{u_{j}}\left(w_{i j}\right)\right\|_{2}\left\|J_{u_{j} v_{i}}\left(v_{i}\right)\right\|_{2} \\
& \leq \kappa\left(u_{j}\right)\left\|\nabla f_{u_{j}}-\nabla p_{u_{j}}\right\|_{C\left(N_{u_{j}} \cap B_{u_{j}}\right)},
\end{aligned}
$$

and

$$
\begin{aligned}
\left\|\hat{\sigma}_{v_{i}}-\sigma_{v_{i}} J_{v_{i} \xi}\left(v_{i}\right)\right\|_{2} & \leq \frac{1}{n+1} \sum_{j=0}^{n} \kappa(\xi) \kappa\left(u_{j}\right)\left\|\nabla f_{u_{j}}-\nabla p_{u_{j}}\right\|_{C\left(N_{u_{j}} \cap B_{u_{j}}\right)} \\
& \leq \kappa^{2} \max _{v \in \mathcal{V}}\left\|\nabla f_{v}-\nabla p_{v}\right\|_{C\left(N_{v} \cap B_{v}\right)} .
\end{aligned}
$$

Combining the above inequalities, we get the desired estimate.

\section{$7 \quad C^{2}$-Manifolds Embedded in $\mathbb{R}^{3}$}

In this section we examine the case when $\Omega$ is an arbitrary compact 2dimensional $C^{2}$-manifold embedded in $\mathbb{R}^{3}$. Our main task is to show how to construct an atlas for $\Omega$ that satisfies the smoothness assumptions of Section 3.1. More details on our method for scattered data fitting on surfaces, including extensive numerical tests, can be found in [9]. Throughout this section, we write $\langle\cdot, \cdot\rangle$ for the usual inner product in $\mathbb{R}^{3}$, and $\|a\|_{2}$ for the Euclidean norm of any 3 -vector $a$.

\subsection{Projection atlas}

Since $\Omega$ is embedded in $\mathbb{R}^{3}$, it can be represented locally as a regular level surface of a $C^{2}$ function of three variables. More precisely, each point $\xi \in \Omega$ has a neighborhood $\mathcal{G}_{\xi}$ in $\mathbb{R}^{3}$ such that $\mathcal{G}_{\xi} \cap \Omega=F_{\xi}^{-1}(0)$, where $F_{\xi}: \mathcal{G}_{\xi} \rightarrow \mathbb{R}$ is a $C^{2}$ function with nonzero gradient $\nabla F_{\xi}$ everywhere in $\mathcal{G}_{\xi} \cap \Omega$, see [19]. Then $n_{\xi}:=\nabla F_{\xi}(\xi) /\left\|\nabla F_{\xi}(\xi)\right\|_{2}$ is a normal vector to $\Omega$ at $\xi$. Moreover, the 
tangent plane $\Gamma_{\xi}$ is the unique plane in $\mathbb{R}^{3}$ that contains $\xi$ and is orthogonal to $n_{\xi}$. Clearly, for all $\zeta \in \mathcal{G}_{\xi} \cap \Omega$, a normal vector to $\Omega$ at $\zeta$ can also be computed as $\nabla F_{\xi}(\zeta) /\left\|\nabla F_{\xi}(\zeta)\right\|_{2}$. It coincides with either $n_{\zeta}$ or $-n_{\zeta}$. Clearly, $\left\langle n_{\xi}, \nabla F_{\xi}(\zeta)\right\rangle>0$ for all $\zeta \in \mathcal{G}_{\xi} \cap \Omega$.

We are now ready to define an atlas associated with $\Omega$. For each $\xi \in \Omega$, let $U_{\xi}$ be the connected component of the open set $\left\{\zeta \in \Omega:\left\langle n_{\xi}, n_{\zeta}\right\rangle \neq 0\right\}$ that contains $\xi$. Then $U_{\xi}$ is an open neighborhood of $\xi$. Clearly, the orthogonal projection $\pi_{\xi}: U_{\xi} \rightarrow \Gamma_{\xi}$ defined by

$$
\pi_{\xi}(\zeta)=\zeta+\left\langle\xi-\zeta, n_{\xi}\right\rangle n_{\xi}, \quad \zeta \in U_{\xi},
$$

is invertible. Assuming that $\gamma_{\xi}^{[1]}, \gamma_{\xi}^{[2]}$ are orthogonal unit vectors in $\Gamma_{\xi}$ such that $\gamma_{\xi}^{[1]} \times \gamma_{\xi}^{[2]}=n_{\xi}$, we can also write

$$
\pi_{\xi}(\zeta)=\xi+\left\langle\zeta-\xi, \gamma_{\xi}^{[1]}\right\rangle \gamma_{\xi}^{[1]}+\left\langle\zeta-\xi, \gamma_{\xi}^{[2]}\right\rangle \gamma_{\xi}^{[2]}
$$

Define $\phi_{\xi}$ by the formula

$$
\phi_{\xi}(\zeta):=\left[\left\langle\zeta-\xi, \gamma_{\xi}^{[1]}\right\rangle,\left\langle\zeta-\xi, \gamma_{\xi}^{[2]}\right\rangle\right]^{T}, \quad \zeta \in U_{\xi} .
$$

We call $\Phi=\left\{U_{\xi}, \phi_{\xi}\right\}_{\xi \in \Omega}$ the projection atlas associated with $\Omega$. The remainder of this subsection is devoted to a proof that $\Phi$ satisfies the hypotheses of Section 3.1.

Theorem 7.1. The projection atlas $\Phi$ is an admissible atlas for $\Omega$, where a basic covering $\left\{\tilde{U}_{\xi}\right\}_{\xi \in \Omega}$ is given by any open neighborhoods of $\xi$ such that the closure of $\tilde{U}_{\xi}$ is a compact set contained in $U_{\xi}$.

Proof. By the choice of $U_{\xi}, \phi_{\xi}$ is invertible. Consider the coordinate system for $\mathbb{R}^{3}$ with coordinate vectors $\gamma_{\xi}^{[1]}, \gamma_{\xi}^{[2]}, n_{\xi}$ and origin $\xi$. For any $\mu \in U_{\xi}$, the equation $F_{\mu}=0$ determines an implicit function $x^{[3]}=\delta_{\mu}\left(x^{[1]}, x^{[2]}\right)$ in a neighborhood of $\phi_{\xi}(\mu)$, such that

$$
\phi_{\xi}^{-1}\left(x^{[1]}, x^{[2]}\right)=\xi+x^{[1]} \gamma_{\xi}^{[1]}+x^{[2]} \gamma_{\xi}^{[2]}+\delta_{\mu}\left(x^{[1]}, x^{[2]}\right) n_{\xi} .
$$

Since $\left\langle n_{\xi}, \nabla F_{\mu}(\mu)\right\rangle=\left\langle n_{\xi}, n_{\mu}\right\rangle\left\|\nabla F_{\mu}(\mu)\right\|_{2} \neq 0$, the implicit function theorem implies that $\delta_{\mu}\left(x^{[1]}, x^{[2]}\right)$ is a $C^{2}$ function in a neighborhood of $\phi_{\xi}(\mu)$. Assuming $\mu \in U_{\xi} \cap U_{\zeta}$, we also have

$$
\phi_{\zeta \xi}\left(x^{[1]}, x^{[2]}\right)=\left(\phi_{\zeta} \circ \phi_{\xi}^{-1}\right)\left(x^{[1]}, x^{[2]}\right)=\left[\phi_{\zeta \xi}^{[1]}\left(x^{[1]}, x^{[2]}\right), \phi_{\zeta \xi}^{[2]}\left(x^{[1]}, x^{[2]}\right)\right]^{T},
$$


where for $i=1,2$,

$$
\phi_{\zeta \xi}^{[i]}\left(x^{[1]}, x^{[2]}\right)=\left\langle\xi-\zeta+x^{[1]} \gamma_{\xi}^{[1]}+x^{[2]} \gamma_{\xi}^{[2]}+\delta_{\mu}\left(x^{[1]}, x^{[2]}\right) n_{\xi}, \gamma_{\zeta}^{[i]}\right\rangle
$$

in a neighborhood of $\phi_{\xi}(\mu)$. Therefore $\phi_{\zeta \xi}: \phi_{\xi}\left(U_{\xi} \cap U_{\zeta}\right) \rightarrow \phi_{\zeta}\left(U_{\xi} \cap U_{\zeta}\right)$ is a $C^{2}$ mapping.

For later use, we now obtain explicit formulas for the Jacobian $J_{\zeta \xi}(\xi):=$ $J\left(\phi_{\zeta \xi}\right)\left(\phi_{\xi}(\xi)\right)$ as defined in Section 3.1, and its determinant. By the above construction, the implicit function $x^{[3]}=\delta_{\xi}\left(x^{[1]}, x^{[2]}\right)$ is $C^{2}$ in a neighborhood of the origin $\phi_{\xi}(\xi)$. Moreover, it vanishes together with its gradient at the origin. Hence, by (7.1),

$$
J_{\zeta \xi}(\xi)=\left[\left\langle\gamma_{\zeta}^{[i]}, \gamma_{\xi}^{[j]}\right\rangle\right]_{i, j=1,2}
$$

Clearly, the determinant of this matrix is the projection of $n_{\zeta}=\gamma_{\zeta}^{[1]} \times \gamma_{\zeta}^{[2]}$ on $n_{\xi}$, i.e.,

$$
\operatorname{det} J_{\zeta \xi}(\xi)=\left\langle n_{\zeta}, n_{\xi}\right\rangle
$$

It remains to show that the charts $\left(U_{\xi}, \phi_{\xi}\right)$ depend smoothly on $\xi$ in the sense of Definition 3.1. To this end, we fix $\xi \in \Omega$ and let $\tilde{U}_{\xi} \subset U_{\xi}$ be an open neighborhood of $\xi$ such that the closure of $\tilde{U}_{\xi}$ is a compact set contained in $U_{\xi}$. Then $\inf _{\mu \in \tilde{U}_{\xi}}\left|\left\langle n_{\xi}, n_{\mu}\right\rangle\right|>0$, and hence $\left\langle n_{\zeta}, n_{\mu}\right\rangle \neq 0$ for all $\mu \in \tilde{U}_{\xi}$ and all $\zeta$ in some neighborhood of $\xi$. This implies that $\tilde{U}_{\xi} \subset U_{\zeta}$ for all $\zeta$ sufficiently close to $\xi$.

For any $\zeta \in U_{\xi}$, we define a coordinate system in $\Gamma_{\zeta}$ with origin $\zeta$ and orthonormal coordinate vectors $\tilde{\gamma}_{\zeta}^{[1]}, \tilde{\gamma}_{\zeta}^{[2]}$, where

$$
\begin{gathered}
\tilde{\gamma}_{\zeta}^{[1]}=\hat{\gamma}_{\zeta}^{[1]} /\left\|\hat{\gamma}_{\zeta}^{[1]}\right\|_{2}, \quad \hat{\gamma}_{\zeta}^{[1]}=\gamma_{\xi}^{[1]}-\left\langle\gamma_{\xi}^{[1]}, \nabla F_{\xi}(\zeta)\right\rangle \nabla F_{\xi}(\zeta) \\
\tilde{\gamma}_{\zeta}^{[2]}=\tilde{n}_{\zeta} \times \tilde{\gamma}_{\zeta}^{[1]}, \quad \tilde{n}_{\zeta}=\nabla F_{\xi}(\zeta) /\left\|\nabla F_{\xi}(\zeta)\right\|_{2} .
\end{gathered}
$$

Set

$$
\tilde{\phi}_{\zeta}(\mu):=\left[\left\langle\mu-\zeta, \tilde{\gamma}_{\zeta}^{[1]}\right\rangle,\left\langle\mu-\zeta, \tilde{\gamma}_{\zeta}^{[2]}\right\rangle\right]^{T}, \quad \mu \in \tilde{U}_{\xi} \subset U_{\zeta}
$$

Since the coordinate system $\gamma_{\zeta}^{[1]}, \gamma_{\zeta}^{[2]}$ can be obtained from $\tilde{\gamma}_{\zeta}^{[1]}, \tilde{\gamma}_{\zeta}^{[2]}$ by an orthogonal linear transformation $r_{\zeta}: \mathbb{R}^{2} \rightarrow \mathbb{R}^{2}$,

$$
\tilde{\phi}_{\zeta}=r_{\zeta} \circ \phi_{\zeta}
$$

Since $F_{\xi}$ is a $C^{2}$ function and $\nabla F_{\xi}(\zeta) \neq 0$ for all $\zeta \in U_{\xi}$, it follows that $\tilde{\phi}_{\zeta}(\mu)$, as a function of $\zeta$, is continuously differentiable at $\zeta=\xi$. 
Finally, for a fixed $\mu \in \tilde{U}_{\xi}$ consider $M(\zeta):=J_{\mu}\left(r_{\zeta} \circ \phi_{\zeta}\right)(\mu)=J\left(r_{\zeta}\right) J_{\zeta \mu}(\mu)$. In view of $(7.2)$,

$$
M(\zeta)=J\left(r_{\zeta}\right)\left[\left\langle\gamma_{\zeta}^{[i]}, \gamma_{\mu}^{[j]}\right\rangle\right]_{i, j=1,2}=\left[\left\langle\tilde{\gamma}_{\zeta}^{[i]}, \gamma_{\mu}^{[j]}\right\rangle\right]_{i, j=1,2}
$$

which is continuously differentiable at $\zeta=\xi$, as required.

\subsection{Projected gradients}

Let $f \in C^{1}(\Omega)$, and let $f_{\xi}=f \circ \phi_{\xi}^{-1}$. Since $\Omega$ is embedded in $\mathbb{R}^{3}$, the gradient $\nabla f_{\xi}=\left(\frac{\partial f_{\xi}}{\partial x^{[1]}}, \frac{\partial f_{\xi}}{\partial x^{[2]}}\right)$ of $f_{\xi}$ can be identified with the 3 -vector

$$
\operatorname{grad} f_{\xi}=\frac{\partial f_{\xi}}{\partial x^{[1]}} \gamma_{\xi}^{[1]}+\frac{\partial f_{\xi}}{\partial x^{[2]}} \gamma_{\xi}^{[2]}
$$

lying in the tangent plane $\Gamma_{\xi} \subset \mathbb{R}^{3}$. Adopting a notation similar to that in Section 3.2, we write

$$
\operatorname{grad}_{\xi} f(\mu):=\left(\operatorname{grad} f_{\xi}\right)\left(\phi_{\xi}(\mu)\right), \quad \mu \in U_{\xi},
$$

for the gradient of $f_{\xi}$ evaluated at $\phi_{\xi}(\mu)$. We call $\operatorname{grad}_{\xi} f(\mu)$ the projected gradient of $f$ at $\mu$. It is easy to see that $\operatorname{grad}_{\xi} f(\xi)$ coincides with the standard gradient of a function on a 2 -surface in $\mathbb{R}^{3}$, as defined for example in [33, p. 96]. We also need projected gradients when $\mu \neq \xi$.

Lemma 7.2. For any $\xi \in \Omega$ and $\zeta \in U_{\xi}$, the projected gradient $\operatorname{grad}_{\zeta} f(\zeta)$ is the orthogonal projection of $\operatorname{grad}_{\xi} f(\zeta)$ onto $\Gamma_{\zeta}$. In particular,

$$
\operatorname{grad}_{\zeta} f(\zeta)=\operatorname{grad}_{\xi} f(\zeta)-\left\langle\operatorname{grad}_{\xi} f(\zeta), n_{\zeta}\right\rangle n_{\zeta},
$$

and

$$
\operatorname{grad}_{\xi} f(\zeta)=\operatorname{grad}_{\zeta} f(\zeta)-\frac{\left\langle\operatorname{grad}_{\zeta} f(\zeta), n_{\xi}\right\rangle}{\left\langle n_{\zeta}, n_{\xi}\right\rangle} n_{\zeta}, \quad \text { if }\left\langle n_{\zeta}, n_{\xi}\right\rangle \neq 0
$$

where $n_{\zeta}$ and $n_{\xi}$ are the unit normal vectors to $\Gamma_{\zeta}$ and $\Gamma_{\xi}$, respectively.

Proof. We have

$$
\operatorname{grad}_{\xi} f(\zeta)=\frac{\partial f_{\xi}}{\partial x^{[1]}}\left(\phi_{\xi}(\zeta)\right) \gamma_{\xi}^{[1]}+\frac{\partial f_{\xi}}{\partial x^{[2]}}\left(\phi_{\xi}(\zeta)\right) \gamma_{\xi}^{[2]}
$$


Its projection onto $\Gamma_{\zeta}$ is therefore

$$
\begin{aligned}
& \left(\frac{\partial f_{\xi}}{\partial x^{[1]}}\left(\phi_{\xi}(\zeta)\right)\left\langle\gamma_{\xi}^{[1]}, \gamma_{\zeta}^{[1]}\right\rangle+\frac{\partial f_{\xi}}{\partial x^{[2]}}\left(\phi_{\xi}(\zeta)\right)\left\langle\gamma_{\xi}^{[2]}, \gamma_{\zeta}^{[1]}\right\rangle\right) \gamma_{\zeta}^{[1]} \\
& +\left(\frac{\partial f_{\xi}}{\partial x^{[1]}}\left(\phi_{\xi}(\zeta)\right)\left\langle\gamma_{\xi}^{[1]}, \gamma_{\zeta}^{[2]}\right\rangle+\frac{\partial f_{\xi}}{\partial x^{[2]}}\left(\phi_{\xi}(\zeta)\right)\left\langle\gamma_{\xi}^{[2]}, \gamma_{\zeta}^{[2]}\right\rangle\right) \gamma_{\zeta}^{[2]}
\end{aligned}
$$

This last expression coincides with $\operatorname{grad}_{\zeta} f(\zeta)$, since

$$
\nabla_{\zeta} f(\zeta)=\nabla_{\xi} f(\zeta) J_{\xi \zeta}(\zeta)=\nabla_{\xi} f(\zeta)\left[\left\langle\gamma_{\xi}^{[i]}, \gamma_{\zeta}^{[j]}\right\rangle\right]_{i, j=1,2}
$$

by (3.2) and (7.2).

The formulas (7.4) and (7.5) for the projection and inverse projection, respectively, follow immediately.

\subsection{Consistent triangulations}

As mentioned in Section 3.4, every compact 2-dimensional smooth manifold $\Omega$ admits a triangulation $\mathcal{T}$. Let $\Omega$ be embedded into $\mathbb{R}^{3}$, and let $\Phi$ be the projection atlas for it. For any $\varepsilon>0$ there is a triangulation $\mathcal{T}$ of $\Omega$ consistent with $\Phi$ and with the mesh size $h<\varepsilon$. See e.g. [2] for a construction of suitable triangulations using sufficiently dense samples of points on 2-dimensional manifolds embedded in $\mathbb{R}^{3}$.

\subsection{Interpolation and data fitting}

Using projected gradients, we can reformulate the interpolation problem

$$
s_{\mathcal{T}}(v)=a_{v}, \quad \nabla_{v} s_{\mathcal{T}}(v)=\sigma_{v}, \quad \text { all } v \in \mathcal{V},
$$

of $(4.1)$ as

$$
s_{\mathcal{T}}(v)=a_{v}, \quad \operatorname{grad}_{v} s_{\mathcal{T}}(v)=c_{v}, \quad \text { all } v \in \mathcal{V},
$$

where $c_{v}=\sigma_{v}^{[1]} \gamma_{\xi}^{[1]}+\sigma_{v}^{[2]} \gamma_{\xi}^{[2]}$ is a vector in $\Gamma_{v}$.

An advantage of the formulation (7.7) over (7.6) is that each $c_{v}$ is determined by just three real numbers (the Cartesian coordinates of $c_{v}$ ), whereas $\sigma_{v}$ requires two real numbers and the tangent vectors $\gamma_{\xi}^{[1]}, \gamma_{\xi}^{[2]}$. Thus, when defining $s_{\mathcal{T}}$ by $(7.7)$, we do not need any coordinate systems in the tangent planes $\Gamma_{v}$. 
Clearly, Algorithms 4.1 and 5.1 can now be formulated without reference to any coordinate systems in the tangent planes provided we use projected gradients. Assume the data is given as $\left\{a_{v}, c_{v}\right\}_{v \in \mathcal{V}}$, where $a_{v}$ are real numbers and $c_{v}$ are 3 -vectors in $\Gamma_{v}$. Then to construct a $C^{1}$ function $s_{\mathcal{T}}$ defined on $\Omega$ that satisfies the interpolation conditions (7.7), we simply apply Algorithm 4.1, where we replace the formula for $g_{i}$ in Step 3 by

$g_{i}:=c_{v_{i}}-\frac{\left\langle c_{v_{i}}, n_{\xi}\right\rangle}{\left\langle n_{v_{i}}, n_{\xi}\right\rangle} n_{v_{i}}$, and require in Step 4 that $s_{\xi}$ interpolates the values $\left\{a_{v_{i}}\right\}_{i=1}^{3}$ and the gradients corresponding to the tangent vectors $\left\{g_{i}\right\}_{i=1}^{3}$ at the vertices $\left\{w_{i}\right\}_{i=1}^{3}$. Algorithm 5.1 describing the first stage of the two-stage data fitting method can also be reformulated by replacing the vectors $\sigma_{v, v_{i}}$ in Step $1 \mathrm{~d}$ by $c_{v, v_{i}}:=\operatorname{grad} p_{v}\left(\tilde{v}_{i}\right)-\left\langle\operatorname{grad} p_{v}\left(\tilde{v}_{i}\right), n_{v_{i}}\right\rangle n_{v_{i}}$ for $i=0, \ldots, n$, and by taking the average of $c_{v_{i}, v}$ 's instead of $\sigma_{v_{i}, v}$ 's in Step 2. Precise formulations can be found in [9].

Theorems 4.3 and 6.1 give error bounds for our interpolation and scattered data fitting methods, respectively, in terms of the mesh size $h$ of $\mathcal{T}$, i.e., the length of the longest edge in the triangles in the set $\left\{\triangle_{\xi}\right\}_{\xi \in \Omega}$, the smallest angle $\alpha$ appearing in the triangles in this set, and the parameter $\kappa$ defined in (6.1). In the case of surfaces embedded in $\mathbb{R}^{3}$, there are more convenient parameters to play the role of $h, \alpha, \kappa$. Let us define the mesh size $\tilde{h}$ as the maximum distance in $\mathbb{R}^{3}$ between any pair of vertices $v \in \mathcal{V}$ connected in $\mathcal{T}$. By actually connecting these pairs of vertices by straight line segments, we obtain a 2-dimensional triangulation in $\mathbb{R}^{3}$. Let $\tilde{\alpha}$ be the smallest angle appearing in its triangles. Let, furthermore, $\tilde{\kappa}(\xi)$ be the maximum of $\left\langle n_{\xi}, n_{v}\right\rangle^{-1}$ over all $v \in \mathcal{V} \cap U_{\xi}$ such that $\xi=\phi_{\xi}(\xi)$ belongs to the closure of a triangle of $\triangle_{\xi}$ attached to $\phi_{\xi}(v)$, and let $\tilde{\kappa}=\max _{\xi \in \Omega} \tilde{\kappa}(\xi)$. Assuming $\Phi$ is the projection atlas, it is not difficult to see that (a) $h \leq \tilde{h}$, (b) $\tilde{\alpha}>0$ implies $\alpha>0$ if $\tilde{h}$ is sufficiently small, and (c) $\kappa<\infty$ if and only if $\tilde{\kappa}<\infty$. (Note that (c) follows from (3.1), (7.2) and (7.3).) Thus, in the case of the projection atlas, Theorems 4.3 and 6.1 can be reformulated with $\tilde{h}, \tilde{\alpha}, \tilde{\kappa}$ in place of $h, \alpha, \kappa$, see [9].

\section{Remarks}

Remark 8.1. The problem of fitting functions defined on surfaces arises in many applications, see for example $[1,3,4,5,11,12,14,15,16,22,23,28$, $29,32,34]$, and references therein. Used parametrically, such functions can be applied to the problem of modelling surfaces of arbitrary topological type 
from point clouds, see for example [17, 18, 35].

Remark 8.2. Many of the papers mentioned in the above remark deal with the sphere in $\mathbb{R}^{3}$. For a survey of interpolation and scattered data fitting methods on the sphere, see [14]. For some specific methods, see [16, 22, 23, $28,32,34]$.

Remark 8.3. The method of this paper is closely related to work of Demjanovich $[11,12]$. He also computes an interpolant $s$ at a point $\xi$ on the manifold by using local charts $\left(U_{\xi}, \phi_{\xi}\right)$ and finite element interpolation in $\phi_{\xi}\left(U_{\xi}\right)$. A key difference is that for each evaluation point $\xi$, his method involves interpolation of the original function values and derivatives assigned to certain points in $\phi_{\xi}\left(U_{\xi}\right)$ determined by the finite element scheme, whereas in our methods we only interpolate projected gradients corresponding to the vertices of the underlying triangulation $\mathcal{T}$, compare Steps 3 and 4 of Algorithm 4.1. Therefore, our interpolation operator only requires function values and gradients at the vertices of $\mathcal{T}$, which makes it possible to design a two-stage scattered data fitting method. Only one of the methods in $[11,12]$ (based on interpolation with Courant hat functions) has similar properties for general manifolds, but it does not produce a $C^{1}$ interpolant.

Remark 8.4. The special case of our method for surfaces in $\mathbb{R}^{3}$ (Section 7) is also closely related to work of Pottmann [29], which also makes use of projected gradients. (It is not difficult to see that equation (7.5) describes the $\pi$-transform of [29].) However, instead of using local approximation methods to estimate gradients, he constructs a kind of minimum norm network.

Remark 8.5. Here we have made use of the standard bivariate $C^{1}$ quadratic Powell-Sabin macro-element to solve the interpolation problem in the tangent plane. Its key feature is that it is constructed from only nine pieces of data, the values and gradients at the three vertices of the macro-triangle. Using the same data, we can also construct an interpolant based on the classical $C^{1}$ reduced Clough-Tocher macro-element. It is based on a split of the macrotriangle into three subtriangles (typically using the barycenter), and is a cubic polynomial on each piece. Along each edge its cross derivative is restricted to be a linear polynomial. Yet another possibility is a modified quadratic Powell-Sabin macro-element on a 12-split [30], where the cross derivatives are assumed linear rather than piecewise linear on the edges of the macrotriangles. Note that with either the Clough-Tocher or Powell-Sabin-12 macroelement, the assumption (3.4) will not be needed. 
Remark 8.6. Instead of the Powell-Sabin macro-element, bivariate interpolation techniques of a completely different nature (and not necessarily based on triangulations) could in principle be combined with the mapping into planes via charts satisfying Definition 3.1. For that to work, the interpolation techniques must meet certain requirements, in particular they must be invariant with respect to the shifts and rotations of the interpolation centers, and must depend smoothly on the data and the location of the centers. It is easy to see that one of the requirements is that the domain of influence of each center is compact. Indeed, otherwise the value of the interpolating function at a given point may be discontinuous with respect to the centers that are at the boundary of $U_{\xi}$.

Remark 8.7. It follows from the Poincaré-Hopf index theorem [26] that any continuous tangent vector field on a compact differentiable manifold without boundary vanishes at a point unless the Euler number of the manifold is zero. Recall that the Euler number of an oriented surface of genus $g$ is $2-2 g$. Thus, the Euler number is zero for the torus, but is nonzero for surfaces of other topological types, including the sphere. For the sphere, this is just the "hairy ball' theorem which states that there is no nonvanishing continuous tangent vector field on the sphere, and explains why we need to use local rotations $r_{\zeta}$ in Definition 3.1. Indeed, if the charts $\left(U_{\xi}, \phi_{\xi}\right)$ for $\Omega$ can be found such that Definition 3.1 holds with $r_{\zeta}$ being the identity in all cases, then one can easily construct a smooth tangent vector field on $\Omega$ by taking unit vectors corresponding to partial derivatives of all local parametrizations. Then the Poincaré-Hopf theorem implies that the Euler number of $\Omega$ is zero, which is a severe restriction on the topology of the manifold.

Remark 8.8. By the Whitney immersion theorem, any 2-dimensional manifold can be immersed in $\mathbb{R}^{3}$. Clearly, the projection atlas can be used on these immersions, where the correct tangent planes have to be chosen for the points of self-intersection. For any 2-dimensional $C^{2}$-submanifold of $\mathbb{R}^{n}$, $n>3$, arguing as in Section 7.1, we can use local orthogonal projections on tangent planes to define an admissible atlas in the sense of Definition 3.1. This construction is also applicable to arbitrary 2-dimensional $C^{2}$-manifolds in view of the Whitney embedding theorem, which says that any smooth 2-dimensional manifold can be smoothly embedded into $\mathbb{R}^{4}$.

Remark 8.9. For the sake of simplicity, in this paper we consider only compact 2-dimensional manifolds without boundary. Clearly, our method is local, 
and therefore can be used on non-compact manifolds that have a countable basis for their topology. Indeed, by a theorem of Radó, such manifolds can be triangulated such that every point has a neighborhood that meets only finitely many triangles [24].

Remark 8.10. The method is also applicable to manifolds with boundary. The main theoretical results of this paper will hold if we assume that all points on the boundary of $\Omega$ are either vertices of the triangulation $\mathcal{T}$ or edge points with respect to it, see Section 3.4. In this case Definition 3.2 needs obvious adjustments for the points $\xi$ on the boundary of $\Omega$, requiring that $\phi_{\xi}(\xi)$ is on the boundary of $P_{\xi}$ rather than in its interior. Another approach could be to introduce a unique $C^{1}$ extension of the Powell-Sabin spline beyond the boundary of a triangulation. This would then allow interior points on the boundary of $\Omega$ and work with the projection atlas as in Section 7 .

Remark 8.11. To extend our method to higher dimensional manifolds, one would need a construction of local $C^{1}$ interpolants in $n$ variables, with $n>2$, completely determined by the function and gradient values at vertices, and depending smoothly on this data and the vertex locations. Similarly, to obtain $C^{2}$ or higher smoothness $C^{r}$ with our scheme, a local bivariate $C^{r}$ interpolant determined by the function and gradient values at vertices is needed. Recall that all known macro-elements of higher smoothness [21] require higher order derivative values.

Remark 8.12. If $\Omega$ is the 2 -dimensional sphere $\mathbb{S}^{2}$, then an admissible atlas $\left\{\left(U_{\xi}, \phi_{\xi}\right)\right\}_{\xi \in \mathbb{S}^{2}}$ can be defined using either central or stereographic projections onto the tangent planes rather than the orthogonal projections as in Section 7.1. The central projection has the property that the edge points with respect to any consistent triangulation are segments of great circles on $\mathbb{S}^{2}$. The advantage of the stereographic projection is that $U_{\xi}$ can be chosen to be $\mathbb{S}^{2} \backslash\{-\xi\}$ provided $-\xi$ is the center of the stereographic projection that defines $\phi_{\xi}$. Therefore, very coarse triangulations, for example the one defined by a tetrahedron inscribed in the sphere, are consistent with the atlas.

Remark 8.13. In the case when $\Omega$ is the torus $\mathbb{T}^{2}$, the following simple atlas is admissible. The torus with inner radius $R-r$ and outer radius $R+r$ is defined parametrically by the equations

$$
\begin{aligned}
& x=(R+r \cos v) \cos u \\
& y=(R+r \cos v) \sin u, \\
& z=r \sin v,
\end{aligned}
$$


with $u, v \in[0,2 \pi)$. For each $\xi$ on the torus, let $u_{\xi}, v_{\xi}$ be its parameter values. We can reparametrize the torus as

$$
\begin{aligned}
& x=\left(R+r \cos \left(v_{\xi}+v\right)\right) \cos \left(u_{\xi}+u\right), \\
& \left.y=\left(R+r \cos \left(v_{\xi}+v\right)\right)\right) \sin \left(u_{\xi}+u\right), \\
& \left.z=r \sin \left(v_{\xi}+v\right)\right),
\end{aligned}
$$

with $u, v \in[-\pi, \pi)$, and define the chart $\left(U_{\xi}, \phi_{\xi}\right)$ by setting $\phi_{\xi}(\zeta)=(u, v) \in$ $\mathbb{R}^{2}$, where $u, v$ are the parameter values of $\zeta$, and letting $U_{\xi}$ be the set of all points $\zeta$ with $\phi_{\xi}(\zeta) \in(-\pi, \pi)^{2}$. This family of charts depends smoothly on $\xi$ in the sense of Definition 3.1, where no local transformations $r_{\zeta}$ are needed. Moreover, the transition mappings $\phi_{\zeta} \circ \phi_{\xi}^{-1}$ are the shifts $(u, v) \mapsto$ $\left(u+u_{\xi}-u_{\zeta}, v+v_{\xi}-v_{\zeta}\right)$. Hence, all Jacobians $J_{\zeta \xi}$ are unit matrices, which makes the transformations in Step 3 of Algorithm 4.1 and in Step 1(d) of Algorithm 5.1 trivial. Note that using this atlas with our method is equivalent to interpreting the data on $\mathbb{T}^{2}$ as periodic data on $\mathbb{R}^{2}$, constructing a periodic triangulation, and interpolating or approximating the data by the ordinary Powell-Sabin spline.

Acknowledgement. We would like to thank anonymous referees for helpful suggestions.

\section{References}

[1] P. Alfeld, M. Neamtu and L. L. Schumaker, Fitting scattered data on sphere-like surfaces using spherical splines, J. Comp. Appl. Math. 73 (1996), 5-43.

[2] N. Amenta and M. W. Bern, Surface reconstruction by Voronoi filtering, Discr. Comput. Geom. 22 (1999), 481-504.

[3] R. E. Barnhill and T. A. Foley, Methods for constructing surfaces on surfaces, in "Geometric Modeling: Methods and Their Applications", Springer, Berlin, 1991, pp. 1-15.

[4] R. E. Barnhill, B. R. Piper and S. E. Stead, A multidimensional surface problem: pressure on a wing, Comput. Aided Geom. Design 2 (1985), 185-187. 
[5] R. E. Barnhill and H. S. Ou, Surfaces defined on surfaces, Comput. Aided Geom. Design 7 (1990), 323-336.

[6] W. Dahmen, R. H. J. Gmelig Meyling and J. H. M. Ursem, Scattered data interpolation by bivariate $C^{1}$-piecewise quadratic functions, $A p$ prox. Theory \& its Appl. 6:3 (1990), 6-29.

[7] O. Davydov, R. Morandi and A. Sestini, Local hybrid approximation for scattered data fitting with bivariate splines, Comput. Aided Geom. Design 23 (2006), 703-721.

[8] O. Davydov, A. Sestini and R. Morandi, Local RBF approximation for scattered data fitting with bivariate splines, in "Trends and Applications in Constructive Approximation," (M. G. de Bruin, D. H. Mache, and J. Szabados, Eds.), ISNM Vol. 151, Birkhäuser, 2005, pp. 91-102.

[9] O. Davydov and L. L. Schumaker, Scattered data fitting on surfaces using projected Powell-Sabin splines, in "Mathematics of Surfaces XII", to appear.

[10] O. Davydov and F. Zeilfelder, Scattered data fitting by direct extension of local polynomials to bivariate splines, Advances in Comp. Math. 21 (2004), 223-271.

[11] Yu. K. Demjanovich, Construction of spaces of local functions on manifolds, Metody Vychisl. (1985) no. 14, 100-109.

[12] Yu. K. Demjanovich, Local approximations of functions given on manifolds, Amer. Math. Soc. Transl. (2) 159 (1994), 53-76.

[13] Yu. K. Demjanovich, Spline approximations on manifolds, Int. J. Wavelets, Multiresolution and Information Processing 4 (2006), 383403.

[14] G. Fasshauer and L. L. Schumaker, Scattered data fitting on the sphere, in "Mathematical Methods for Curves and Surfaces II", M. Dæhlen, T. Lyche, and L. L. Schumaker (eds.), Vanderbilt University Press, Nashville, 1998, 117-166.

[15] T. A. Foley, D. A. Lane, G. M. Nielson, R. Franke, and H. Hagen, Interpolation of scattered data on closed surfaces, Comput. Aided Geom. Design 7 (1990), 303-312. 
[16] W. Freeden, Spherical spline interpolation-basic theory and computional aspects, J. Comp. Appl. Math. 11 (1985), 367-375.

[17] C. M. Grimm and J. F. Hughes, Modeling surfaces of arbitrary topology using manifolds, in "Proceedings of SIGGRAPH 95," 1995, pp. 359-368

[18] Xianfeng Gu, Ying He, and Hong Qin, Manifold splines, in "Proceedings of ACM Symposium on Solid and Physical Modeling," 2005, pp. 27-38.

[19] M. W. Hirsch, Differential Topology, Springer-Verlag, 1976.

[20] L. Ch. Kinsey, Topology of Surfaces, Springer, 1993.

[21] M.-J. Lai and L. L. Schumaker, Spline Functions on Triangulations, Cambridge University Press, Cambridge, 2007.

[22] C. L. Lawson, $C^{1}$ surface interpolation for scattered data on a sphere, Rocky Mountain J. Math. 14 (1984), 177-202.

[23] T. Lyche and L. L. Schumaker, A multiresolution tensor spline method for fitting functions on the sphere, SIAM J. Sci. Computing 22 (2000), $724-746$.

[24] W. S. Massey, Algebraic Topology: An Introduction, Springer, 1977.

[25] C. R. F. Maunder, Algebraic Topology, Van Nostrand Reinhold Company, London, 1970.

[26] J. W. Milnor, Topology from the Differential Viewpoint, Princeton University Press, 1965.

[27] M. Neamtu and L. L. Schumaker, On the approximation order of splines on spherical triangulations, Adv. in Comp. Math. 21 (2004), 3-20.

[28] G. M. Nielson and R. Ramaraj, Interpolation over a sphere based upon a minimum norm network, Comput. Aided Geom. Design 4 (1987), 41-58.

[29] H. Pottmann, Interpolation on surfaces using minimum norm networks, Computer Aided Geometric Design 9 (1992), 51-67.

[30] M. J. D. Powell and M. A. Sabin, Piecewise quadratic approximations on triangles, ACM Trans. Math. Software 3 (1977), 316-325. 
[31] L. L. Schumaker, Dual bases for spline spaces on cells, Computer Aided Geometric Design 5 (1988), 277-284.

[32] L. L. Schumaker and C. Traas, Fitting scattered data on spherelike surfaces using tensor products of trigonometric and polynomial splines, Numer. Math. 60 (1991), 133-144.

[33] J. A. Thorpe, Elementary Topics in Differential Geometry, Springer, 1979.

[34] G. Wahba, Spline interpolation and smoothing on the sphere, SIAM J. Sci. Stat. Computing 2 (1981), 5-16.

[35] L. Ying and D. Zorin, A simple manifold-based construction of surfaces of arbitrary smoothness, ACM Transactions on Graphics 23 (2004), 271275 . 